\title{
El practicum como herramienta de evaluación de las competencias profesionales de los alumnos del Máster de Contabilidad y Auditoría
}

\author{
The practicum as assessment tool for professional competences in \\ the Master degree of Accounting and Auditing
}

\author{
Estíbaliz Biedma López (estibaliz.biedma@uca.es) \\ Nieves Gómez Aguilar (nieves.gomez@uca.es) \\ Emiliano Ruiz Barbadillo (emiliano.ruiz@uca.es) \\ Universidad de Cádiz (España) \\ http://dx.doi.org/10.12795/EDUCADE.2011.i02.07
}

\begin{abstract}
RESUMEN: El objetivo de este trabajo es proponer herramientas de evaluación de las competencias profesionales que deben ser adquiridas por los alumnos del Máster de Contabilidad y Auditoría para su inserción profesional. Concretamente, analizaremos el potencial del practicum (prácticas externas insertas en el plan de estudios del título) como medio de evaluar dichas competencias. Existen numerosos estudios que ponen de manifiesto cuáles son las competencias profesionales a adquirir en el entorno de la contabilidad y la auditoría. No obstante, estas competencias tienen entre sus características la dificultad de su evaluación en el aula.

El practicum supone un entorno ideal para la puesta en práctica y evaluación de las mismas dado que se trata del mejor escenario de simulación previo a su inserción laboral. Para ello, hemos seleccionado aquellas competencias profesionales más adecuadas a la inserción laboral de los alumnos del Máster y hemos elaborado materiales que ayuden al desarrollo y evaluación del practicum en función de dichas competencias.

Según este planteamiento, al utilizar estos materiales los tutores internos (profesores), los tutores externos (tutores en la empresa) y los alumnos están, de manera implícita, desarrollando y evaluando estas competencias.

La siguiente fase es llevar a cabo un análisis de la adecuación de estos materiales a través de la técnica de cuestionarios dirigidos a las partes implicadas. La aportación fundamental de este estudio es la generación para la comunidad universitaria de materiales eficaces para la evaluación de estas competencias profesionales en el ámbito de la docencia de postgrado de Contabilidad y Auditoría.
\end{abstract}

PALABRAS CLAVE: Competencias profesionales, Auditoría, Docencia en Contabilidad, Evaluación.

\begin{abstract}
The aim of this work is to propose tools for the evaluation of professional competences which should be acquired by students of the Master in Accounting and Auditing for their insertion in the labour market. Specially, we analyse the potential of the practicum (internships which form part of the syllabus of the Master) as a means to evaluate these competences.

Many existing studies point out the professional competences that should be acquired in the field of accounting and auditing. Nonetheless, these competences are inherently difficult to be assessed in the lecture hall.

The practicum is an ideal context to the development and evaluation of these competences, due to it being the best simulated setting before actually beginning their working career. With this in mind, we have selected those professional competences which are most propitious for their labour insertion.
\end{abstract}

Experiencia docente. Recibido: 12-01-11 - Versiones revisadas: 14-03-11, Aceptado: 14-06-11

Licencia Creative Commons (CC) BY-NC-ND · Asociación Española de Contabilidad y Administración de Empresas - AECA 
We go on to draw up material which helps students, internal tutors (academics) and external tutors (company tutors) to develop and evaluate these key competences. In a subsequent stage we analyse the idealness if this material by means of questionnaires administer to tutors. The main contribution of this study is to put at the disposal of the university community effective material for the development and evaluation of professional competences.

KEYWORDS: Professional competences, Auditing, Accounting and Auditing teaching, Evaluation.

\section{INTRODUCCIÓN}

En los últimos años, el debate sobre cómo mejorar el proceso enseñanza-aprendizaje a nivel internacional ha destacado la necesidad de que la formación universitaria haga un mayor esfuerzo en otorgar al estudiante de herramientas y recursos que le hagan más capaces de desarrollar con éxito una labor profesional acorde a su perfil. A fin de otorgar a las universidades un papel más relevante en la capacitación de los estudiantes para lograr su mejor desempeño en los puestos de trabajo se ha realzado la importancia de la formación basada en competencias (UNESCO, 1998). Esto implica que los profesores universitarios deberán asumir el reto de formar a futuros profesionales, de forma que su asignatura será una parte de un conjunto de conocimientos, habilidades y actitudes que deberá ser trasvasado y desarrollado por el estudiante durante su vida académica.

Entre los organismos más cercanos a la disciplina contable, la International Federation of Accountants (IFAC, 2010) trata de fomentar la formación basada en competencias como herramienta para crear mejores profesionales. Así, define competencia como la capacidad de una persona para ejecutar un trabajo cumpliendo con un determinado estándar en un entorno laboral (IFAC, 2010: 23); entendiendo por capacidad el conjunto de conocimientos, habilidades y actitudes, valores y ética profesionales requeridos para demostrar competencia (IFAC, 2010: 22).

En este sentido, numerosos estudios e informes han propuesto listas de competencias profesionales que deben poseer los egresados de títulos relacionados con la Contabilidad y Auditoría. Si bien existe un gran consenso con respecto a dichas propuestas, hay un aspecto relacionado con la formación en este tipo de competencias que consideramos que no está del todo resuelto y es su evaluación.

Así, en un entorno de aulas como es el académico en el que no se pueden recrear determinados tipo de situaciones (jerarquía, equipos multidisciplinares de trabajo, ...), con el tiempo a dedicar limitado a las horas de clases, así como con profesores no formados específicamente en esas competencias el desarrollo de dichas competencias así como su evaluación resulta muy compleja.

Es por ello que el practicum, en aquellos títulos que lo poseen, puede aportar un entorno ideal para el desarrollo y evaluación de estas competencias transversales, sobre todo, porque el estudiante puede practicarlas en un entorno similar a aquél en el que después deberá demostrar que las posee. Además, tiene más tiempo para desarrollarlas ya que normalmente un practicum tiene una duración mínima de dos meses. Como ventaja adicional, el encargado de supervisar el desarrollo de esas competencias y su posterior evaluación, que denominaremos tutores de empresa, suelen ser aquéllos que después valorarán dicha formación a la hora de decidir una posible contratación futura.

Si bien la formación en competencias profesionales a través del practicum ofrece estas tres ventajas, también tiene sus inconvenientes ya que hay que contar con la diversidad de empresas o entidades que participan en un practicum, la dedicación 
de los tutores de empresas, las distintas líneas de formación que se pueden plantear, la a veces difícil comunicación entre tutores internos y externos. Para intentar minimizar estos inconvenientes, es muy útil diseñar materiales para los evaluadores de estas competencias que les guíen y les ayuden en la evaluación de las mismas.

El objetivo que proponemos en este trabajo es evaluar la utilidad de los materiales que se han propuesto a los evaluadores (tutores académicos y de empresa) del practicum del Máster en Contabilidad y Auditoría de la Universidad de Cádiz. Concretamente, para el tutor académico se ha diseñado un Modelo de Memoria de prácticas a cumplimentar por el alumno (ver Anexo 1) y un cuestionario para la evaluación del practicum (ver Anexo 2). La Memoria de prácticas realizada por el alumno ha ido destinada a recoger evidencia documental tanto del conocimiento adquirido por el alumno en el practicum, como de la actividad realizada en la empresa en dicho periodo. Por su parte, el cuestionario de evaluación del practicum cumplimentado por el tutor académico ha estado orientado a recabar información sobre el seguimiento y cumplimiento por parte del alumno durante el practicum, además del nivel alcanzado por éste en aquellas competencias profesionales que han podido ser observadas por el tutor académico durante las reuniones de tutorización. Para que el tutor externo plasme los resultados del desempeño del alumno en el puesto de trabajo en términos de competencias profesionales se ha diseñado otro cuestionario de evaluación (ver Anexo 3),

El planteamiento general ha sido que la utilización de estos materiales, además orientar la evaluación del nivel de competencia profesional alcanzado por el alumno en el practicum, de manera implícita servirá de guía a los tutores para conocer qué competencias deben adquirir los alumnos durante el proceso, y por tanto, promover el desarrollo de las mismas.

Una vez terminado el proceso de evaluación de los estudiantes del máster, al objeto de evaluar la utilidad de los materiales utilizados, hemos desarrollado y enviado unos cuestionarios a los tutores implicados en dicha evaluación con la intención de recabar su opinión sobre (1) la propuesta de competencias profesionales o trasversales que se han debido evaluar en el practicum y (2) si los materiales aportados les han sido de utilidad en dicho proceso (ver Anexos 4 y 5). La muestra objeto de análisis se refiere a los tutores externos e internos de los 30 alumnos del practicum de dicho Máster.

Los resultados obtenidos muestran que tanto los tutores internos como los externos consideran la propuesta de competencias profesionales a evaluar en practicum como muy adecuada, aunque en su mayoría consideran insuficiente los materiales aportados como guía para evaluar dichas competencias.

\section{MARCO TEÓRICO}

La sociedad actual se caracteriza fundamentalmente por su capacidad de cambio continuo, debido principalmente a la tecnificación, que agiliza vertiginosamente la puesta en práctica de los descubrimientos científicos, y a la evolución y acumulación del conocimiento, que imposibilita abarcar el conocimiento de un campo específico (Dirección General de Empleo y Consejo Social de la Universidad de Cádiz, 2010).

En un entorno tan complejo y cambiante como el actual y con tanta presión competitiva, los profesionales inmersos en el mundo empresarial no sólo deben tener los conocimientos adecuados, sino que también debe poseer habilidades profesionales, valores y actitudes que les capaciten para adaptarse de forma continua al cambio. En concreto, la IFAC ha señalado que los profesionales contables actuales además de adquirir conocimientos técnicos y destrezas contables (competencias específicas), necesitan desarrollar habilidades que les permitan ser 
buenos asesores empresariales, analistas financieros, buenos comunicadores, negociadores capaces y directivos de primera, y al mismo tiempo, mantener un alto nivel de conducta ética y valores profesionales (IFAC, 2009, p. 38). Son estas habilidades profesionales las que van a dotar al experto contable de la capacidad para aplicar los conocimientos, los valores y la ética profesional al contexto laboral, convirtiéndolo así en un profesional competente.

Todo este conjunto de capacidades, habilidades y actitudes complementarias a la formación técnica forma parte de lo que se viene denominando como competencias profesionales ${ }^{1}$, que no sólo consideran el componente de conocimiento profesional (saber), sino que va más allá incluyendo habilidades profesionales (saber hacer), valores, ética y actitudes profesionales (saber ser o estar).

Por tanto, adquirir competencias o trabajar en competencias implica la búsqueda de aprendizajes más globales y prácticos, donde los nuevos conocimientos estén dirigidos al desarrollo de la capacidad de actuación de los sujetos, que les permita poder llevar a cabo procesos de acción cada vez más complejos (Zabalza, 2004).

La posesión de competencias profesionales se está convirtiendo en un factor determinante de los procesos de selección de las empresas. En esta línea, la Unión Europea ha manifestado que de los casi siete millones de nuevos puestos de trabajo que se generarán en los próximos 10 años, la mayoría exigirán niveles altos de conocimiento y de competencias, por lo que señala como aspecto clave la formación en capacidades y habilidades. Lejos de crear perfiles profesionales concretos, se aboga por profesionales con competencias que encajen en labores diferentes y que permitan que la vida laboral del ciudadano vaya cambiando según varíe el mercado laboral (El País, 12 de Abril de 2010).

En esta línea, tal y como apunta Zabalza (2004), las competencias profesionales suelen combinar lo contextual (al tratarse de demandas de formación que adquieren su sentido en función de un contexto determinado) y lo general, en tanto que incluyen la condición de generalizables. Así pues, la clasificación más utilizada en el diseño de los nuevos grados universitarios considera, no sólo las competencias específicas (definidas en función del perfil académico-profesional que reclame la sociedad en que se han de insertar), sino también la necesaria presencia de competencias genéricas o transversales, comunes a la mayoría de profesiones y que se relacionan con la puesta en práctica integrada de aptitudes, conocimientos, y valores adquiridos, por lo que se requieren en diversas áreas ocupacionales (Solanes et al., 2008).

Para adquirir estos conocimientos, habilidades profesionales, actitudes y valores de forma satisfactoria, la IFAC manifiesta que en todo programa formativo debe coexistir un periodo de educación formal con un periodo de experiencia práctica (International Education Practice Statement, IEPS, 3). Este periodo de experiencia práctica previo a su inserción laboral (conocido también como practicum) permite al estudiante no sólo profundizar en la adquisición de conocimientos, habilidades profesionales y actitudes, sino también demostrar la adquisición de dichas capacidades, es decir, demostrar que es competente. En este sentido, para Ia IFAC (2010: 23) la competencia está ligada a la ejecución del trabajo, mientras que aplican

\footnotetext{
${ }^{1}$ La Ley Orgánica 5/2002 de 19 de junio, de las Cualificaciones y de la Formación Profesional entiende por competencia profesional el conjunto de conocimientos y capacidades que permiten el ejercicio de la actividad profesional conforme a las exigencias de la producción y el empleo. Sin embargo, algunos de los libros blancos definen el concepto de competencia profesional exclusivamente como una competencia específica ligada al saber hacer, distinguiéndolo así de los conocimientos disciplinares que aportan el saber y de las competencias académicas (Libro Blanco del Título de Grado en Economía y en Empresa).
} 
el concepto de capacidad o capacidades al conjunto atributos (conocimientos profesionales, habilidades profesionales y valores, ética y actitudes profesionales) mantenidos por los individuos que les van a permitir desempeñar sus funciones profesionales.

Es decir, la competencia implica demostrar que se posee la habilidad de desempeñar una tarea al estándar de actuación requerido y debe ser constatable en la práctica, mediante comportamientos evidenciables (Villa y Poblete, 2004). ${ }^{2}$

El contexto de la práctica representa por tanto el entorno más adecuado para poder adquirir y evaluar algunas de estas competencias profesionales, que no siempre se pueden adquirir y evaluar a través de la educación formal desarrollada en los cursos académicos.

Tal y como señala Tejada (2005), la evaluación de las competencias, por sus propias características e implicaciones, es una de las tareas más importantes del proceso de formación, en general, y en el practicum en particular. Esta evaluación no debe utilizarse sólo para determinar quién posee ciertas competencias, sino también servir de guía para el aprendizaje promoviéndolas entre los estudiantes (Cano, 2008). Por ello, se hace necesaria la articulación de dispositivos válidos y fiables donde se pueda evidenciar que se poseen las competencias profesionales requeridas, conocidos por todos los participantes en el practicum (tutor académico, tutor en la empresa y alumno), y especialmente por el alumno, de manera que se pueda orientar su aprendizaje. Existen diversos instrumentos que permiten inferir el nivel de competencia profesional adquirida a través del desempeño en el prácticum. La utilización de unos u otros dependerá del tipo de desempeño, así como de la cantidad y calidad de la evidencia que debe recogerse (Tejada, 2005).

Tejada (2005, p. 22) distingue tres tipos de evidencias:

- Evidencias sobre el conocimiento, que pueden ser evaluables a través de pruebas teórico y prácticas. En esta línea la IFAC (2010) en el International Education Standard $n^{\circ} 5$ (IES 5: Practical Experience Requirements), establece la necesidad que de la experiencia práctica sea evaluada en base a documentación escrita revisada por el tutor académico y exposiciones orales por parte de los alumnos en prácticas.

- Evidencias sobre el proceso, que corresponden a aquellos elementos que indican la calidad en la ejecución de una tarea. Para este tipo de evidencias propone como instrumento la observación y el análisis dentro del proceso de trabajo. En este punto es fundamental la participación del tutor en la empresa.

- Evidencias del producto, que corresponden a los resultados o productos identificables y tangibles, y que pueden usarse como referentes para demostrar que una actividad fue realizada.

En base a lo expuesto, la experiencia que describe este trabajo se marcó como objetivo la creación de herramientas que orienten la adquisición de determinadas competencias profesionales que debían ser obtenidas por los alumnos del Máster de Contabilidad y Auditoría de la Universidad de Cádiz para su inserción profesional, y permitan su evaluación.

\footnotetext{
2 No obstante, la IFAC (2010: 22) afirma que la posesión de capacidades supone un buen indicio de que los individuos tienen el potencial para actuar de forma competente en el lugar de trabajo.
} 
A este respecto, se diseñaron las siguientes fuentes informativas con la intención de obtener suficiente base documental para los tres tipos de evidencias señaladas por Tejada (2005):

- La memoria del practicum elaborada por el estudiante en el marco del Trabajo fin de Máster (ver Anexo 1).

- La defensa oral de la memoria del practicum.

- El Informe de evaluación de competencias del tutor en la empresa (ver Anexo 3).

- Un informe del tutor académico sobre el seguimiento y cumplimiento por parte del estudiante durante el periodo de prácticas (ver Anexo 2).

La pregunta de investigación que nos planteamos es si estos materiales elaborados han resultado útiles para la adquisición y evaluación de determinadas competencias profesionales.

\section{DESCRIPCIÓN DE LA EXPERIENCIA}

\subsection{ENTORNO DE APLICACIÓN}

Para llevar a cabo el objetivo propuesto en este trabajo, utilizamos como entorno de análisis el Módulo de Aplicación del Máster en Contabilidad y Auditoría impartido como Máster oficial de la Universidad de Cádiz. Este máster está estructurado en tres módulos. Los dos primeros módulos corresponden al periodo de educación formal de trabajo en el aula con el profesor, durante el cual se impartieron diversas materias en relación con los conocimientos básicos que debe adquirir un profesional contable. ${ }^{3} \mathrm{El}$ tercer módulo lo compone un "Módulo de Aplicación" constituido por el practicum (prácticas en Empresas o Instituciones), que tiene asignados 14 créditos ECTS de los 60 que forman el Máster en su totalidad. Además de estos 14 créditos, en el Módulo de aplicación se contemplan 6 destinados a la elaboración y presentación del Trabajo Fin de Máster.

\subsection{Perfil de LOS estudiantes}

El número de alumnos que ha realizado el Máster es 33. De los cuales sólo han intervenido en esta experiencia 30, ya que los otros tres optaron no realizaron el practicum al optar por el perfil de investigador. Los datos descriptivos más significativos del colectivo sujeto al estudio se recogen en la Tabla 1.

Como puede verse en la Tabla 1, las titulaciones de procedencia están claramente relacionadas con el Máster, aunque un $30 \%$ de los alumnos las habían acabado hace más de 5 años. Esto último explica que la mitad de los alumnos ya tengan experiencia laboral, por lo que es de esperar que los niveles de partida en competencias profesionales en estos casos sean bastante altos. Entre aquéllos que no acreditan haber tenido un contrato laboral previo, 4 han realizado prácticas en empresas durante el desarrollo de sus titulaciones anteriores, por lo que de alguna forma también habrán podido desarrollar alguna de las competencias profesionales relacionadas con el Máster.

\footnotetext{
3 Durante cada uno de los cursos, además de trabajar la adquisición de conocimientos profesionales, se trabajó el desarrollo de diversas competencias profesionales específicas y genéricas, a través de diversas metodologías docentes.
} 
Estíbaliz Biedma, Nieves Gómez, Emiliano Ruiz

El practicum como herramienta de evaluación

TABLA 1: DesCRIPCIÓN DE lOS ALUMNOS

\begin{tabular}{|c|c|c|}
\hline \multirow{2}{*}{ Sexo } & Hombre & 12 \\
\hline & Mujer & 18 \\
\hline \multirow{5}{*}{ Titulación de procedencia } & LADE & 15 \\
\hline & Licenciatura C. Económicas y Empresariales & 2 \\
\hline & Licenciado en Economía & 1 \\
\hline & Diplomado en Empresariales & 11 \\
\hline & Doble Diplomatura Turismo y Empresariales & 1 \\
\hline \multirow{10}{*}{$\begin{array}{l}\text { Año de finalización de la titulación } \\
\text { anterior }\end{array}$} & 2009 & 9 \\
\hline & 2008 & 10 \\
\hline & 2007 & 2 \\
\hline & 2005 & 1 \\
\hline & 2004 & 1 \\
\hline & 2003 & 2 \\
\hline & 2002 & 2 \\
\hline & 2000 & 1 \\
\hline & 1999 & 1 \\
\hline & 1997 & 1 \\
\hline \multirow{2}{*}{ Universidad de titulación anterior } & Cádiz & 25 \\
\hline & Otras & 5 \\
\hline \multirow{3}{*}{ Experiencia profesional anterior } & Prácticas en empresas & 4 \\
\hline & Contrato laboral & 15 \\
\hline & Ninguna & 11 \\
\hline
\end{tabular}

\subsection{Perfil de Las entidades}

En la Tabla 2 hacemos una breve descripción de las empresas que han contribuido a la formación práctica de los alumnos del Máster. Sólo mostramos datos de 27 empresas porque 3 de ellas acogieron dos alumnos.

TABLA 2: TAMAÑO Y ACTIVIDAD DE LAS ENTIDADES

\begin{tabular}{|c|c|c|}
\hline \multirow{6}{*}{ Tamaño de la entidad } & Menos de 5 & 7 \\
\hline & Entre 5 y 10 & 6 \\
\hline & Entre 11 y 50 & 7 \\
\hline & Entre 51 y 100 & 5 \\
\hline & Entre 101 y 500 & 1 \\
\hline & Más de 500 & 1 \\
\hline \multirow{11}{*}{$\begin{array}{l}\text { Actividad principal de } \\
\text { la entidad }\end{array}$} & Consultoría y Auditoría & 16 \\
\hline & Artes gráficas e impresión & 1 \\
\hline & Bodegas & 2 \\
\hline & Educación & 1 \\
\hline & Gestión de la investigación & 1 \\
\hline & Gestión tributaria & 1 \\
\hline & Gestión y construcción inmobiliaria & 1 \\
\hline & Gestión medioambiental & 1 \\
\hline & Distribución de productos alimenticios & 1 \\
\hline & Atención a la discapacidad & 1 \\
\hline & Nuevas tecnologías & 1 \\
\hline \multirow{4}{*}{$\begin{array}{l}\text { Forma jurídica de la } \\
\text { entidad }\end{array}$} & Sociedades mercantiles & 21 \\
\hline & Fundaciones & 1 \\
\hline & $\begin{array}{l}\text { Administraciónes públicas o empresas vinculadas a } \\
\text { administraciones públicas }\end{array}$ & 3 \\
\hline & Entidades sin ánimo de lucro & 2 \\
\hline
\end{tabular}


Como podemos ver, dos terceras partes de ellas tienen menos de 50 trabajadores y el $48 \%$ tienen menos de 10 . Esto se explica porque la mayor parte de las prácticas se han realizado en asesorías y firmas de auditoría que en Cádiz se suelen organizar en pequeños despachos profesionales con uno o dos socios y personal de apoyo. Concretamente, el $60 \%$ de las empresas o entidades tienen como actividad habitual el prestar este tipo de servicios.

Debido a la diversidad de empresas, los objetivos formativos de los alumnos han sido muy diversos, así como el lugar de desempeño de la práctica. Esto ha hecho especialmente interesante la elaboración del cuestionario de evaluación del tutor externo, ya que ha tenido que ser lo suficientemente comprensivo para incluir distintas líneas de trabajo y a la vez sintético para no exigir mucho tiempo en su respuesta.

\subsection{Desarrollo}

\section{Fase 1: Selección de las competencias profesionales}

El primer paso en esta experiencia fue la discusión y posterior selección de las competencias profesionales que debían ser desarrolladas por los alumnos del Máster en Contabilidad y Auditoría durante el periodo de prácticas.

Entre los distintos enfoques a la hora de establecer las competencias ${ }^{4}$ nos decantamos por partir de la percepción profesional, donde un grupo de profesores, responsables académicos, empleadores, etc. generan ellos mismos una lista de comportamientos que entienden constituyen actuaciones características de los profesionales eficaces de ése sector. Las actuaciones incluidas en la lista se van agrupando, matizando y reajustando hasta configurar un grupo de competencias sobre la que existe consenso (Zabalza, 2004).

En este sentido partimos de cuatro fuentes de información:

- Las conclusiones del "Proyecto de implantación de un programa de formación y certificación de competencias para titulados universitarios" desarrollado por el Consejo Social y la Dirección General de Empleo de la Universidad de Cádiz (2010). Como resultado de este proyecto un panel de expertos formada por miembros de la Universidad de Cádiz, representantes de empresas e instituciones de la provincia y consultores independientes elaboró un mapa de competencias profesionales fundamentales clasificadas por área de conocimiento.

Este panel clasificó estas competencias profesionales en cuatro categorías: Área de recursos humanos, área de conocimiento, área organizacional y área de compromiso. Así, para el área de conocimiento que denominaron "Económicas" establecieron las siguientes competencias profesionales: Trabajo en equipo, Capacidad de comunicación, Orientación al cliente y Negociación, dentro de las competencias pertenecientes al área de recursos humanos; Compromiso, Iniciativa, Identificación con la compañía y Confianza en sí mismo, dentro de la categoría de Compromiso; Aprendizaje continuo, Adaptabilidad al cambio y Creatividad, dentro del área de conocimiento; y Capacidad de gestión, Organización y planificación y Orientación al logro y resultados, dentro del área organizacional.

- Habilidades profesionales requeridas por la IFAC (2010) para los profesionales contables y auditores, a través del International Accounting Education Standards Board (IAESB) en las guías (International Education Standards, IES) elaboradas sobre los elementos esenciales que debe contener la educación de los profesionales

\footnotetext{
4 Véase Zabalza (2004) o Houston (1990, pag. 2724).
} 
contables. En estas guías entre otros aspectos, además de precisar los conocimientos necesarios, se definen las habilidades, valores, ética, actitudes y experiencia profesional que deben poseer para ser profesionales competentes (IES 3: Professional Skills and General Education; IES 4: Professional Values, Ethics and Attitudes; IES 8: Competence Requirements for Audit Professionals).

- Competencias profesionales reflejadas en la memoria oficial del Máster en Contabilidad y Auditoría de la Universidad de Cádiz ${ }^{5}$. Este Programa Oficial de Postgrado se plantea como objetivo general formar especialistas en Contabilidad, que puedan llevar a cabo diferentes funciones en el ámbito empresarial relacionadas con la dirección contable-financiera, la asesoría contable y la auditoría.

- Análisis de las competencias profesionales destacadas por diversos estudios empíricos como demandadas para los profesionales contables (véase entre otros, Arquero, 2000; Arquero et al., 2009; Kavanagh y Drennan, 2008).

Tras la revisión de estos documentos, teniendo en cuenta la naturaleza del Máster dividido en dos módulos fundamentales de conocimiento (Contabilidad y Auditoría) y la posible diversidad de puestos desempeñados por los alumnos en las prácticas dentro de estas áreas de conocimiento, creímos más conveniente, como punto de partida de esta experiencia, centrarnos como competencias básicas a desarrollar en el practicum en las competencias transversales a buena parte de las actuaciones posibles en este contexto. Seleccionamos algunas de las competencias transversales más demandas para el desempeño profesional en los informes y estudios revisados, prestando especial atención a aquellas sobre las cuales se requería una mayor intensidad del desempeño para los profesionales contables y auditores:

TABLA 3: CoMpetenCIAS PROFESIONALES GENÉRICAS A DESARROLLAR EN EL PRACTICUM DEL MÁSTER DE CONTABILIDAD Y AUDITORÍA DE LA UNIVERSIDAD DE CÁDIZ

\begin{tabular}{|l|}
\hline Identificación y resolución de problemas \\
\hline Compromiso con el trabajo \\
\hline Trabajo en equipo \\
\hline $\begin{array}{l}\text { Indagar e investigar, pensar de forma lógica y analítica, con poder de razonamiento y } \\
\text { análisis crítico. }\end{array}$ \\
\hline Aplicar los conocimientos en la práctica \\
\hline Iniciativa \\
\hline Aprendizaje autónomo \\
\hline Adaptación a nuevas situaciones \\
\hline Análisis y síntesis \\
\hline Comunicación oral y escrita \\
\hline Compromiso por la calidad \\
\hline
\end{tabular}

\section{Fase 2: Elaboración del material para los tutores del practicum}

En la Tabla 4 se muestran los materiales puestos a disposición tanto del tutor interno como del externo para ayudar al desarrollo y evaluación de las competencias profesionales, así como las competencias profesionales que se pretenden incentivar y evaluar con cada uno de los materialesb:

\footnotetext{
5 Véase http://posgrado.uca.es/web/info master.php?id=152\&curso=2010/11.

6 Como puede observarse en los cuestionarios de los Anexos 2 y 3, cada una de las competencias pretende ser inferida a través de una serie de indicadores.
} 
TABLA 4: COMPETENCIAS PROFESIONALES A TRABAJAR A TRAVÉS DE LOS MATERIALES ELABORADOS

\begin{tabular}{|c|c|}
\hline MATERIAL & COMPETENCIAS PROFESIONALES \\
\hline $\begin{array}{l}\text { Modelo de memoria de } \\
\text { prácticas (ver Anexo 1) }\end{array}$ & $\begin{array}{l}\text { - Análisis y síntesis } \\
\text { - Comunicación oral y escrita } \\
\text { - Pensar de forma lógica y analítica, con poder de razonamiento } \\
\text { - y análisis crítico } \\
\text { - Motivación por la calidad }\end{array}$ \\
\hline $\begin{array}{l}\text { Cuestionario del tutor } \\
\text { interno para la } \\
\text { evaluación del } \\
\text { practicum (ver Anexo 2) }\end{array}$ & $\begin{array}{l}\text { - Compromiso con el trabajo } \\
\text { - Iniciativa } \\
\text { - Aprendizaje autónomo } \\
\text { - Aplicación de los conocimientos en la práctica }\end{array}$ \\
\hline $\begin{array}{l}\text { Cuestionario del tutor } \\
\text { externo para la } \\
\text { evaluación del } \\
\text { practicum (ver Anexo 3) }\end{array}$ & $\begin{array}{l}\text { - Aplicar los conocimientos en la práctica } \\
\text { - Identificación y resolución de problemas } \\
\text { - Compromiso con el trabajo } \\
\text { - Trabajo en equipo } \\
\text { - Indagar e investigar, pensar de forma lógica y analítica, con } \\
\text { - Inoder de razonamiento y análisis crítico } \\
\text { - Aprendizaje autónomo }\end{array}$ \\
\hline
\end{tabular}

\section{Fase 3: Asignación de tutores académicos a cada estudiante}

Para guiar el aprendizaje del alumno tanto en el periodo docente como en el practicum a cada alumno de le asigna un "tutor interno" o tutor académico. Se trata de profesores del departamento de Economía Financiera y Contabilidad con docencia en el Máster, encargados de la orientación y supervisión de los estudiantes, así como de ayudarlos en la adquisición de las competencias. Cada alumno mantiene durante el curso varias tutorías. La primera está destinada a explicar al alumno la metodología general a seguir en el Máster, así como la forma de evaluación, plazos de entrega, etc. El resto van dirigidas tanto a hacer un seguimiento del alumno del trabajo realizado por el alumno en el practicum, como a supervisar la realización por parte del alumno del Trabajo Fin de Máster.

\section{Fase 4: Entrega del material a los tutores internos}

El material elaborado se pone a disposición de los tutores internos para que éstos los utilicen en las tutorías que realizan con sus alumnos y los hagan llegar a los tutores externos que se les asignen en las empresas en las que realicen la práctica dichos alumnos.

\section{Fase 5: Asignación de la empresa y de los tutores externos a cada estudiante}

A cada alumno se le asigna un tutor en la empresa donde está realizando las prácticas (tutor externo o tutor de empresa). La labor fundamental de dicho tutor es dirigir al estudiante hacia la consecución de los objetivos formativos previamente establecidos, y ayudarle en el desarrollo y la demostración de las competencias profesionales.

\section{Fase 6: Determinación de los objetivos formativos de la práctica y entrega del material.}

Previamente al inicio de las prácticas, el tutor interno y el tutor externo establecen los objetivos formativos de la práctica, y el primero hace entrega al segundo del Cuestionario de evaluación del tutor externo. En dicho cuestionario se insta al tutor externo a evaluar al alumno tanto por la capacidad para aplicar los conocimientos adquiridos, como en términos de las competencias profesionales que se pretenden desarrollar en el estudiante. De esta forma, el cuestionario pretende ser para el tutor externo una guía para conocer qué aspectos son los que debe observar en el 
estudiante en prácticas, para posteriormente emitir su valoración sobre el nivel alcanzado en dichas competencias.

\section{Fase 7: Realización de las prácticas}

Entre junio y agosto se desarrollan las prácticas de los alumnos con especial seguimiento de sus tutores internos que velarán por el cumplimiento de los objetivos formativos consensuados con el tutor de la empresa.

\section{Fase 8: Evaluación del estudiante}

La valoración que cada estudiante recibirá en el practicum la emite la Comisión Académica y procede de tres fuentes:

- Valoración emitida por el tutor externo a través del Cuestionario del Tutor Externo para la Evaluación del Practicum. ${ }^{7}$ Esta valoración aporta información procedente de la observación directa del desempeño del estudiante en el puesto de trabajo.

- Valoración emitida por el tutor interno a través del Cuestionario del Tutor Interno para la Evaluación del Practicum. Esta valoración se basa fundamentalmente en las reuniones de seguimiento mantenidas con el estudiante.

- Valoración emitida por un tribunal. El tribunal es designado por la Comisión Académica a propuesta del coordinador del programa y está integrado por tres profesores de entre los que hayan participado en el Máster (queda excluido el tutor del alumno). Este tribunal evalúa al alumno en base a la Memoria de Prácticas depositada y a la exposición oral de dicha memoria.

\section{Fase 9: Evaluación de los materiales}

Una vez terminada la práctica y evaluado el alumno, se inicia la etapa en la que consultamos con los evaluadores del practicum la utilidad de los materiales que han utilizado. Dicho proceso y los resultados alcanzados se describen en los apartados siguientes.

\section{METODOLOGÍA}

Para determinar si los materiales elaborados han resultado útiles para la adquisición y evaluación de determinadas competencias profesionales, hemos recabado y analizado la opinión de aquéllos que han utilizado los materiales en su proceso de evaluación, es decir, los tutores externos y los tutores internos de los alumnos.

Para obtener su opinión, hemos elaborado sendos cuestionarios que, acompañados de una introducción, se han enviado a los distintos tutores. Dichos cuestionarios se adjuntan en el Anexo 4 y 5.

\subsection{OBteNCIÓN DE LA OPINIÓN DEL TUTOR INTERNO}

En el cuestionario enviado al tutor interno, hemos añadido a modo de introducción una referencia al objetivo del cuestionario y hemos enumerado las competencias trasversales objeto de evaluación a través de la práctica realizada y de las memorias presentadas. Las cuestiones planteadas a los tutores internos se han separado en dos bloques principalmente:

\footnotetext{
7 En este sentido Solanes et al. (2008) afirman que el cuestionario es uno de los métodos de evaluación de mayor aplicación en el ámbito laboral.
} 
a) Capacidades profesionales: El objetivo de este bloque de cuestiones es saber qué peso otorgan a las distintas capacidades que se les proponen en relación con el desempeño profesional de un titulado de este Máster.

b) Utilidad de los materiales: En este caso le hemos propuesto que se manifiesten en relación con (1) la utilidad de los materiales como guía para saber qué competencias han de ser evaluadas y (2) la utilidad como herramienta de evaluación de las mismas.

A estos dos bloques se le ha añadido otro con algunas cuestiones relacionadas con las características personales del tutor interno y la posibilidad de añadir algún comentario adicional.

El cuestionario fue enviado por correo electrónico a los 11 profesores que han tutorizado a los alumnos del Máster que han realizado práctica externas, siendo el índice de respuesta del 100\%. La descripción del perfil de los tutores que han contestado al cuestionario se describe en la Tabla 5:

TAbla 5: Perfil del tutor interno

\begin{tabular}{|c|c|c|c|}
\hline \multirow{4}{*}{ Experiencia docente } & $0-10$ años & \multicolumn{2}{|l|}{1} \\
\hline & 11-15 años & \multicolumn{2}{|l|}{2} \\
\hline & 16-20 años & \multicolumn{2}{|l|}{5} \\
\hline & N/D & \multicolumn{2}{|l|}{3} \\
\hline \multirow{6}{*}{ Experiencia laboral } & No & \multicolumn{2}{|l|}{5} \\
\hline & Sí & 6 & \\
\hline & \multirow{2}{*}{$N^{\circ}$ años } & $0-5$ años & 5 \\
\hline & & $6-15$ & 1 \\
\hline & \multirow{2}{*}{ Actividad profesional } & Administración & 3 \\
\hline & & Auditoría y Consultoría & 3 \\
\hline
\end{tabular}

Como se aprecia en la Tabla 5, el número de años de experiencia docente de los tutores internos es bastante elevado, lo que le otorga a su opinión una gran relevancia en base al conocimiento de los sistemas de evaluación que se les presupone. Asimismo, más de la mitad de ellos tienen experiencia laboral en sectores relacionados con el Máster lo que les permitirá una mayor facilidad para formarse una opinión sobre las capacidades transversales trabajadas y evaluadas en un entorno laboral.

\subsection{OBteNCIÓN DE LA OPINIÓN DEL TUTOR EXTERNO}

A su vez, en el cuestionario enviado al tutor externo hemos incluido como introducción una referencia al objetivo del estudio haciendo hincapié en la importancia de la evaluación de las competencias profesionales, manteniendo el resto del cuestionario el mismo formato que el cuestionario del tutor interno.

El cuestionario fue enviado por correo electrónico a los 27 tutores externos, recibiendo 13 respuestas. El perfil de los tutores que han contestado al cuestionario se describe en la Tabla 6: 
TABLA 6: PERFIL DEL TUTOR EXTERNO

\begin{tabular}{|l|l|l|}
\hline \multirow{3}{*}{ Sexo } & Hombre & 8 \\
\cline { 2 - 3 } & Mujer & 5 \\
\hline \multirow{4}{*}{ Exivel de estudios } & Doctor & 1 \\
\cline { 2 - 3 } & Licenciado & 11 \\
\cline { 2 - 3 } & Diplomado & 1 \\
\hline \multirow{5}{*}{ Experiencia laboral } & $0-10$ años & 2 \\
\cline { 2 - 3 } & $11-20$ años & 3 \\
\cline { 2 - 3 } & $21-30$ & 1 \\
\cline { 2 - 3 } & $31-40$ & \\
\hline
\end{tabular}

\section{ANÁLISIS DE RESULTADOS}

En relación con la importancia otorgada por los tutores externos e internos a las capacidades propuestas en esta experiencia, los resultados procedentes de un análisis descriptivo se muestran en las Tablas 7 y 8 . Con relación a los tutores internos, por término medio, todas las capacidades planteadas han sido consideradas importantes para el desempeño adecuado de las tareas propias de un titulado en el Máster de Contabilidad y Auditoría.

TABLA 7. DESCRIPTIVOS DE LA IMPORTANCIA OTORGADA A LAS CAPACIDADES PROFESIONALES SELECCIONADAS: ESTADÍSTICOS

\begin{tabular}{|c|c|c|c|c|c|c|c|c|c|}
\hline CAPACIDADES & & $\mathrm{N}$ & Media & Mediana & Moda & $\begin{array}{c}\text { Desv. } \\
\text { típ. }\end{array}$ & Mín. & Máx. & $\begin{array}{c}\text { U de M- } \\
\text { W } \\
\text { p-valor }\end{array}$ \\
\hline Identificación y resolución & TE & 13 & 6,38 & 7,00 & 7 & 0,77 & 5 & 7 & \\
\hline de problemas & TI & 0 & & & & & & & \\
\hline Comnromico ron ol trahgir & TE & 13 & 6,23 & 7,00 & 7 & 1,17 & 3 & 7 & $0 \cap 33$ \\
\hline 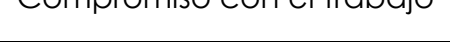 & $\mathrm{TI}$ & 11 & 5,82 & 6,00 & 6 & 0,40 & 5 & 6 & 0.000 \\
\hline & TE & 13 & 5,85 & 6,00 & 7 & 1,14 & 4 & 7 & \\
\hline manajo en equipo & TI & 0 & & & & & & & \\
\hline Indagar e investigar, pensar & TE & 13 & 6,38 & 7,00 & 7 & 0,77 & 5 & 7 & \\
\hline $\begin{array}{c}\text { con poder de razonamiento } \\
\text { y análisis crítico }\end{array}$ & $\mathrm{TI}$ & 11 & 6,18 & 7,00 & 7 & 1,08 & 4 & 7 & 0.820 \\
\hline Aplicar los conocimientos & TE & 13 & 5,54 & 6,00 & $6^{a}$ & 1,51 & 2 & 7 & 303 \\
\hline en la práctica & TI & 11 & 6,18 & 7,00 & 7 & 1,08 & 4 & 7 & J.303 3 \\
\hline Iniciativg & $\mathrm{TE}$ & 13 & 6,54 & 7,00 & 7 & 0,66 & 5 & 7 & $\cap \cap \cap \cap * * *$ \\
\hline IIICTAIIVA & TI & 11 & 4,73 & 5,00 & 4 & 0,79 & 4 & 6 & 0.000 \\
\hline Anrondizain autónomo & TE & 13 & 6,00 & 6,00 & 6 & 0,71 & 5 & 7 & 0106 \\
\hline Apremalzaje dutomomis & $\mathrm{TI}$ & 11 & 5,45 & 5,00 & 5 & 1,13 & 4 & 7 & 0.100 \\
\hline Análicis y cíntecis & TE & 0 & & & & & & & \\
\hline Anallsis y sintesis & $\mathrm{TI}$ & 10 & 6,10 & 6,50 & 7 & 1,20 & 4 & 7 & \\
\hline Comunicación oral vocrita & TE & 0 & & & & & & & \\
\hline comunicacion oral y escilia & $\mathrm{TI}$ & 11 & 5,36 & 5,00 & $4^{a}$ & 1,12 & 4 & 7 & \\
\hline Compromiso por la calidad & TE & 0 & & & & & & & \\
\hline & $\mathrm{Tl}$ & 11 & 5,91 & 6,00 & 6 & 0,70 & 5 & 7 & \\
\hline
\end{tabular}

$\mathrm{TE}=$ Tutor externo, $\mathrm{Tl}=$ Tutor interno. ${ }^{a}$ Existen varias modas y se muestra el menor de los valores. ${ }^{* * *}$ indica un nivel de confianza superior al $99 \%$.

Las opiniones varían entre otorgarles algo de importancia o considerarlas esenciales. Las que por término medio han sido consideradas más importantes son la capacidad 
de Indagar e investigar, pensar de forma lógica y analítica, con poder de razonamiento y análisis crítico, y la capacidad para aplicar los conocimientos adquiridos en la práctica. Respecto a estas capacidades, la mayoría opina que son esenciales. Le sigue en valoración positiva la capacidad de análisis y síntesis, donde la mayoría opina que es muy importante o esencial (80\% de los tutores internos).

TABLA 8. DESCRIPTIVOS DE LA IMPORTANCIA OTORGADA A LAS CAPACIDADES PROFESIONALES SELECCIONADAS: PORCENTAJES DE LAS RESPUESTAS DE LOS TUTORES

\begin{tabular}{|c|c|c|c|c|c|c|c|c|c|c|}
\hline \multicolumn{2}{|l|}{ CAPACIDADES } & & 1 & 2 & 3 & 4 & 5 & 6 & 7 & Total \\
\hline \multirow{4}{*}{$\begin{array}{c}\text { Identificación y resolución de } \\
\text { problemas }\end{array}$} & TE & Frecuencia & 0 & 0 & 0 & 0 & 2 & 4 & 7 & 13 \\
\hline & & $\%$ & 0 & 0 & 0 & 0 & 15,4 & 30,8 & 53,8 & 100 \\
\hline & $\mathrm{TI}$ & Frecuencia & & & & & & & & \\
\hline & & $\%$ & & & & & & & & \\
\hline \multirow{4}{*}{ Compromiso con el trabajo } & $\mathrm{TE}$ & Frecuencia & 0 & 0 & 1 & 0 & 1 & 4 & 7 & 13 \\
\hline & & $\%$ & 0 & 0 & 7,7 & 0 & 7,7 & 30,8 & 53,8 & 100 \\
\hline & $\mathrm{TI}$ & Frecuencia & 0 & 0 & 0 & 0 & 2 & 9 & 0 & 11 \\
\hline & & $\%$ & 0 & 0 & 0 & 0 & 18,2 & 81,8 & 0 & 100 \\
\hline \multirow{4}{*}{ Trabajo en equipo } & TE & Frecuencia & 0 & 0 & 0 & 2 & 3 & 3 & 5 & 13 \\
\hline & & $\%$ & 0 & 0 & 0 & 15,4 & 23,1 & 23,1 & 38,5 & 100 \\
\hline & $\mathrm{TI}$ & Frecuencia & & & & & & & & \\
\hline & & $\%$ & & & & & & & & \\
\hline \multirow{4}{*}{$\begin{array}{l}\text { Indagar e investigar, pensar de forma } \\
\text { lógica y analítica, con poder de } \\
\text { razonamiento y análisis crítico }\end{array}$} & TE & Frecuencia & 0 & 0 & 0 & 0 & 2 & 4 & 7 & 13 \\
\hline & & $\%$ & 0 & 0 & 0 & 0 & 15,4 & 30,8 & 53,8 & 100 \\
\hline & $\mathrm{TI}$ & Frecuencia & 0 & 0 & 0 & 1 & 2 & 2 & 6 & 11 \\
\hline & & $\%$ & 0 & 0 & 0 & 9,1 & 18,2 & 18,2 & 54,5 & 100 \\
\hline \multirow{4}{*}{$\begin{array}{c}\text { Aplicar los conocimientos en la } \\
\text { práctica }\end{array}$} & $\mathrm{TE}$ & Frecuencia & 0 & 1 & 0 & 2 & 2 & 4 & 4 & 13 \\
\hline & & $\%$ & 0 & 7,7 & 0 & 15,4 & 15,4 & 30,8 & 30,8 & 100 \\
\hline & $\mathrm{TI}$ & Frecuencia & 0 & 0 & 0 & 1 & 2 & 2 & 6 & 11 \\
\hline & & $\%$ & 0 & 0 & 0 & 9,1 & 18,2 & 18,2 & 54,5 & 100 \\
\hline \multirow{4}{*}{ Iniciativa } & TE & Frecuencia & 0 & 0 & 0 & 0 & 1 & 4 & 8 & 13 \\
\hline & & $\%$ & 0 & 0 & 0 & 0 & 7,7 & 30,8 & 61,5 & 100 \\
\hline & $\mathrm{TI}$ & Frecuencia & 0 & 0 & 0 & 5 & 4 & 2 & 0 & 11 \\
\hline & & $\%$ & 0 & 0 & 0 & 45,5 & 36,4 & 18,2 & 0 & 100 \\
\hline \multirow{4}{*}{ Aprendizaje autónomo } & $\mathrm{TE}$ & Frecuencia & 0 & 0 & 0 & 0 & 3 & 7 & 3 & 13 \\
\hline & & $\%$ & 0 & 0 & 0 & 0 & 23,1 & 53,8 & 23,1 & 100 \\
\hline & TI & Frecuencia & 0 & 0 & 0 & 2 & 5 & 1 & 3 & 11 \\
\hline & & $\%$ & 0 & 0 & 0 & 18,2 & 45,5 & 9,1 & 27,3 & 100 \\
\hline \multirow{4}{*}{ Análisis y síntesis } & TE & Frecuencia & & & & & & & & \\
\hline & & $\%$ & & & & & & & & \\
\hline & $\mathrm{TI}$ & Frecuencia & 0 & 0 & 0 & 2 & 0 & 3 & 5 & 10 \\
\hline & & $\%$ & 0 & 0 & 0 & 20 & 0 & 30 & 50 & 100 \\
\hline \multirow{4}{*}{ Comunicación oral y escrita } & TE & Frecuencia & & & & & & & & \\
\hline & & $\%$ & & & & & & & & \\
\hline & $\mathrm{TI}$ & Frecuencia & 0 & 0 & 0 & 3 & 3 & 3 & 2 & 11 \\
\hline & & $\%$ & 0 & 0 & 0 & 27,3 & 27,3 & 27,3 & 18,2 & 100 \\
\hline \multirow{4}{*}{ Compromiso por la calidad } & $\mathrm{TE}$ & Frecuencia & & & & & & & & \\
\hline & & $\%$ & & & & & & & & \\
\hline & $\mathrm{TI}$ & Frecuencia & 0 & 0 & 0 & 0 & 3 & 6 & 2 & 11 \\
\hline & & $\%$ & 0 & 0 & 0 & 0 & 27,3 & 54,5 & 18,2 & 100 \\
\hline
\end{tabular}

$1=$ ninguna importancia, $2=$ muy poca importancia, $3=$ poca importancia, $4=$ algo importante, $5=$ bastante importante, $6=$ muy importante, $7=$ =esencial, $\mathrm{TE}=$ Tutor externo, $\mathrm{Tl}=$ Tutor interno. 
A la que menos importancia se le ha otorgado en cambio ha sido la iniciativa, donde la mayoría ha considerado que tiene algo (45.5\%) o bastante importancia $(36,4 \%)$, seguida de la capacidad de comunicación oral y escrita.

La que mayor grado de acuerdo ha presentado entre los tutores internos es la utilidad del compromiso con el trabajo, donde la mayoría opina que es muy importante (81.8\%), y un $18.2 \%$ que es bastante importante.

En el caso de los tutores externos, la importancia media atribuida a las capacidades señaladas es mayor, siendo todas consideradas mayoritariamente como muy importantes o esenciales. La competencia que los tutores de empresa consideran más importante es la iniciativa, donde el $61.5 \%$ opina que se trata de característica esenciales. Le siguen en importancia la capacidad de identificación y resolución de problemas y la capacidad de Indagar e investigar, pensar de forma lógica y analítica, con poder de razonamiento y análisis crítico, y el compromiso en el trabajo, también consideradas esenciales o muy importantes por el $53.8 \%$ y el $30.8 \%$, respectivamente. Esto contrasta con las opiniones de los tutores internos, sobre todo en el caso de la iniciativa, donde la prueba de U Mann-Whitney constata la existencia de diferencias significativas entre ambos grupos con un nivel de confianza del $99 \%$.

Por el contrario, a la que menos importancia le atribuyen por término medio los tutores de empresa es a la capacidad de aplicar los conocimientos en la práctica, aunque esta competencia ha sido considerada mayoritariamente muy importante y el nivel de desacuerdo entre tutores externos ha sido superior que en el resto de respuestas.

En relación con la utilidad otorgada a los materiales objeto de estudio como herramienta para orientar a los tutores en el desarrollo de las competencias profesionales seleccionadas en el alumno (véanse Tablas 9 y 10), la mayoría le otorgan al menos algo de utilidad, pero no tanta como cabría esperar. Además, las opiniones son más dispares (rango muy amplio en las respuestas, con frecuencias muy igualadas) habiendo tutores que no les otorgan ninguna utilidad y otros que opinan que son esenciales.

En el caso de los tutores internos, los materiales entregados han sido considerados mayoritariamente bastante o algo útiles para el orientar el desarrollo de la mayoría de las competencias, excepto la iniciativa, para la cual estos materiales resultan mayoritariamente poco útiles. No obstante, para la práctica totalidad de las competencias encontramos entre un 20 o un $40 \%$ de tutores internos que opinan que estos materiales son poco o nada útiles. A la vista de estos resultados, parece que en la función de promover el desarrollo de estas competencias, los materiales no son del todo útiles aunque tampoco desechables, lo que sugiere que la vía de mejora pasa por mejorar la explicación en cuanto al propósito de desarrollo de las competencias profesionales señaladas. En este sentido, algunos tutores internos han comentado que por la simple tenencia de los materiales, algunas de las competencias como el compromiso por la calidad no queda claro que haya que fomentar su desarrollo en el alumno, y sugieren que se acompañen estos materiales de algún tipo de documento donde se expresen los objetivos de la supervisión, las competencias profesionales a desarrollar y evaluar, el modo, etc.

Particularizando en las competencias, los materiales han resultado mayoritariamente bastante útiles para el desarrollo de la capacidad de comunicación oral y escrita y la de compromiso en el trabajo. Le siguen en valoración positiva la capacidad de aplicación de los conocimientos adquiridos a la práctica y la capacidad de análisis y síntesis. En esta primera, el $80 \%$ opina que los materiales son algo, bastante, muy útiles o esenciales, mientras que para la capacidad de análisis y síntesis representan el $70 \%$. 
Estíbaliz Biedma, Nieves Gómez, Emiliano Ruiz El practicum como herramienta de evaluación

TABLA 9. DESCRIPTIVOS DE LA UTILIDAD DE LOS MATERIALES PARA ORIENTAR EL DESARROLLO DE LAS CAPACIDADES PROFESIONALES SELECCIONADAS: ESTADÍSTICOS

\begin{tabular}{|c|c|c|c|c|c|c|c|c|}
\hline CAPACIDADES & & $\mathrm{N}$ & Media & Mediana & Moda & $\begin{array}{l}\text { Desv. } \\
\text { típ. }\end{array}$ & Mín. & Máx. \\
\hline \multirow{2}{*}{$\begin{array}{l}\text { Identificación y resolución de } \\
\text { problemas }\end{array}$} & TE & 10 & 4,70 & 5,00 & $4^{a}$ & 1,64 & 1 & 7 \\
\hline & $\mathrm{TI}$ & 0 & & & & & & \\
\hline \multirow{2}{*}{ Compromiso con el trabajo } & TE & 10 & 4,90 & 5,50 & 6 & 1,73 & 1 & 7 \\
\hline & $\mathrm{TI}$ & 10 & 4,20 & 5,00 & $5^{a}$ & 1,81 & 1 & 6 \\
\hline \multirow{2}{*}{ Trabajo en equipo } & TE & 10 & 4,30 & 4,00 & 4 & 1,57 & 1 & 6 \\
\hline & $\mathrm{Tl}$ & 0 & & & & & & \\
\hline \multirow{2}{*}{$\begin{array}{l}\text { Indagar e investigar, pensar de forma } \\
\text { lógica y analítica, con poder de } \\
\text { razonamiento y análisis crítico }\end{array}$} & TE & 10 & 4,60 & 4,50 & $4^{a}$ & 1,58 & 1 & 6 \\
\hline & $\mathrm{Tl}$ & 10 & 4,20 & 4,00 & 4 & 1,75 & 1 & 7 \\
\hline \multirow{2}{*}{$\begin{array}{c}\text { Aplicar los conocimientos en la } \\
\text { práctica }\end{array}$} & TE & 10 & 4,30 & 4,00 & 4 & 1,42 & 1 & 6 \\
\hline & $\mathrm{TI}$ & 10 & 4,50 & 4,50 & 4 & 1,72 & 1 & 7 \\
\hline \multirow{2}{*}{ Iniciativa } & $\mathrm{TE}$ & 10 & 4,60 & 4,50 & 4 & 1,65 & 1 & 7 \\
\hline & $\mathrm{TI}$ & 10 & 3,20 & 3,00 & 3 & 1,23 & 1 & 5 \\
\hline \multirow{2}{*}{ Aprendizaje autónomo } & TE & 9 & 4,33 & 4,00 & 4 & 1,66 & 1 & 7 \\
\hline & $\mathrm{TI}$ & 10 & 4,00 & 4,00 & 4 & 1,63 & 1 & 7 \\
\hline \multirow{2}{*}{ Análisis y síntesis } & TE & 0 & & & & & & \\
\hline & $\mathrm{TI}$ & 10 & 4,40 & 4,50 & $2^{a}$ & 1,71 & 2 & 7 \\
\hline \multirow[t]{2}{*}{ Comunicación oral y escrita } & TE & 0 & & & & & & \\
\hline & $\mathrm{TI}$ & 10 & 4,80 & 5,00 & 7 & 2,04 & 2 & 7 \\
\hline \multirow{2}{*}{ Compromiso por la calidad } & $\mathrm{TE}$ & 0 & & & & & & \\
\hline & $\mathrm{TI}$ & 10 & 4,00 & 4,00 & 4 & 1,70 & 1 & 6 \\
\hline
\end{tabular}

$\mathrm{TE}=$ Tutor externo, $\mathrm{Tl}=$ Tutor interno. ${ }^{a}$ Existen varias modas y se muestra el menor de los valores.

Las capacidades para las cuales los tutores internos opinan que los materiales han sido menos útiles como guía para su desarrollo son la iniciativa y el aprendizaje autónomo. En el caso de la iniciativa, el 70\% opina que los materiales son poco, muy poco o nada útiles como guía. En el caso del aprendizaje autónomo y del compromiso por la calidad, la baja ponderación media se debe fundamentalmente a la alta dispersión de la respuesta.

En cuanto a los tutores externos, que contaban con el Cuestionario del Tutor externo para la Evaluación del Practicum como herramienta para orientar el desarrollo y evaluación de competencias profesionales, sus opiniones también son dispares respecto al grado de utilidad de este mecanismo para la función de guía. No obstante, por término medio los materiales son mejor valorados para mayoría de las competencias que en el caso de los tutores internos. A primera vista, por término medio el cuestionario resulta muy útil o algo útil para incentivar el desarrollo de la mayoría de las competencias. Sólo un $10 \%$ de los tutores externos opina que el material resulta poco, muy poco o nada útil para guiar el desarrollo de las competencias seleccionadas.

La competencia para la que el cuestionario ha recibido peor valoración es el trabajo en equipo aunque para esta sólo el $20 \%$ opina que el material resulta poco o muy poco útil como guía. 
Estíbaliz Biedma, Nieves Gómez, Emiliano Ruiz

El practicum como herramienta de evaluación

TABLA 10. DeSCRIPTIVOS DE LA UTILIDAD DE LOS MATERIALES PARA ORIENTAR EL DESARROLLO DE LAS CAPACIDADES PROFESIONALES SELECCIONADAS: PORCENTAJES DE LAS RESPUESTAS DE LOS TUTORES

\begin{tabular}{|c|c|c|c|c|c|c|c|c|c|c|}
\hline CAPACIDADES & & & 1 & 2 & 3 & 4 & 5 & 6 & 7 & Total \\
\hline \multirow{4}{*}{$\begin{array}{c}\text { Identificación y resolución de } \\
\text { problemas }\end{array}$} & \multirow{2}{*}{$\mathrm{TE}$} & Frecuencia & 1 & 0 & 0 & 3 & 3 & 2 & 1 & 10 \\
\hline & & $\%$ & 10,0 & 0,0 & 0,0 & 30,0 & 30,0 & 20,0 & 10,0 & 100,0 \\
\hline & \multirow{2}{*}{$\mathrm{TI}$} & Frecuencia & & & & & & & & \\
\hline & & $\%$ & & & & & & & & \\
\hline \multirow{4}{*}{ Compromiso con el trabajo } & \multirow{2}{*}{$\mathrm{TE}$} & Frecuencia & 1 & 0 & 0 & 3 & 1 & 4 & 1 & 10 \\
\hline & & $\%$ & 10,0 & 0,0 & 0,0 & 30,0 & 10,0 & 40,0 & 10,0 & 100,0 \\
\hline & \multirow{2}{*}{$\mathrm{TI}$} & Frecuencia & 1 & 1 & 2 & 0 & 3 & 3 & 0 & 10 \\
\hline & & $\%$ & 10,0 & 10,0 & 20,0 & 0,0 & 30,0 & 30,0 & 0,0 & 100,0 \\
\hline \multirow{4}{*}{ Trabajo en equipo } & \multirow{2}{*}{ TE } & Frecuencia & 0 & 1 & 1 & 4 & 1 & 3 & 0 & 10 \\
\hline & & $\%$ & 0 & 10 & 10 & 40 & 10 & 30 & 0 & 100 \\
\hline & \multirow{2}{*}{$\mathrm{TI}$} & Frecuencia & & & & & & & & \\
\hline & & $\%$ & & & & & & & & \\
\hline \multirow{4}{*}{$\begin{array}{l}\text { Indagar e investigar, pensar de } \\
\text { forma lógica y analítica, con } \\
\text { poder de razonamiento y } \\
\text { análisis crítico }\end{array}$} & \multirow{2}{*}{ TE } & Frecuencia & 1 & 0 & 0 & 4 & 1 & 4 & 0 & 10 \\
\hline & & $\%$ & 10,0 & 0,0 & 0,0 & 40,0 & 10,0 & 40,0 & 0,0 & 100,0 \\
\hline & \multirow{2}{*}{$\mathrm{Tl}$} & Frecuencia & 1 & 1 & 0 & 4 & 2 & 1 & 1 & 10 \\
\hline & & $\%$ & 10,0 & 10,0 & 0,0 & 40,0 & 20,0 & 10,0 & 10,0 & 100,0 \\
\hline \multirow{4}{*}{$\begin{array}{c}\text { Aplicar los conocimientos en la } \\
\text { práctica }\end{array}$} & \multirow{2}{*}{ TE } & Frecuencia & 1 & 0 & 0 & 5 & 2 & 2 & 0 & 10 \\
\hline & & $\%$ & 10,0 & 0,0 & 0,0 & 50,0 & 20,0 & 20,0 & 0,0 & 100,0 \\
\hline & \multirow{2}{*}{$\mathrm{TI}$} & Frecuencia & 1 & 0 & 1 & 3 & 2 & 2 & 1 & 10 \\
\hline & & $\%$ & 10,0 & 0,0 & 10,0 & 30,0 & 20,0 & 20,0 & 10,0 & 100,0 \\
\hline \multirow{4}{*}{ Iniciativa } & \multirow{2}{*}{$\mathrm{TE}$} & Frecuencia & 1 & 0 & 0 & 4 & 2 & 2 & 1 & 10 \\
\hline & & $\%$ & 10,0 & 0,0 & 0,0 & 40,0 & 20,0 & 20,0 & 10,0 & 100,0 \\
\hline & Tt & Frecuencia & 1 & 1 & 5 & 1 & 2 & 0 & 0 & 10 \\
\hline & 11 & $\%$ & 10,0 & 10,0 & 50,0 & 10,0 & 20,0 & 0,0 & 0,0 & 100,0 \\
\hline \multirow{4}{*}{ Aprendizaje autónomo } & \multirow{2}{*}{ TE } & Frecuencia & 1 & 0 & 0 & 5 & 1 & 1 & 1 & 9 \\
\hline & & $\%$ & 11,1 & 0,0 & 0,0 & 55,6 & 11,1 & 11,1 & 11,1 & 100,0 \\
\hline & TI & Frecuencia & 1 & 1 & 0 & 5 & 2 & 0 & 1 & 10 \\
\hline & 11 & $\%$ & 10,0 & 10,0 & 0,0 & 50,0 & 20,0 & 0,0 & 10,0 & 100,0 \\
\hline \multirow{4}{*}{ Análisis y síntesis } & \multirow{2}{*}{$\mathrm{TE}$} & Frecuencia & & & & & & & & \\
\hline & & $\%$ & & & & & & & & \\
\hline & \multirow{2}{*}{ TI } & Frecuencia & 0 & 2 & 1 & 2 & 2 & 2 & 1 & 10 \\
\hline & & $\%$ & 0,0 & 20,0 & 10,0 & 20,0 & 20,0 & 20,0 & 10,0 & 100,0 \\
\hline \multirow{4}{*}{ Comunicación oral y escrita } & TE & Frecuencia & & & & & & & & \\
\hline & IL & $\%$ & & & & & & & & \\
\hline & TI & Frecuencia & 0 & 2 & 1 & 2 & 2 & & 3 & 10 \\
\hline & 11 & $\%$ & 0,0 & 20,0 & 10,0 & 20,0 & 20,0 & 0,0 & 30,0 & 100,0 \\
\hline & TE & Frecuencia & & & & & & & & \\
\hline Compromiso por la calidgd & II & $\%$ & & & & & & & & \\
\hline 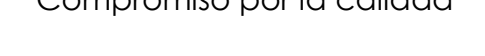 & TI & Frecuencia & 1 & 1 & 1 & 4 & 0 & 3 & 0 & 10 \\
\hline & II & $\%$ & 10,0 & 10,0 & 10,0 & 40,0 & 0,0 & 30,0 & 0,0 & 100,0 \\
\hline
\end{tabular}

$1=$ ninguna utilidad, $2=$ muy poca utilidad, $3=$ poca utilidad, $4=$ algo útil, $5=$ bastante útil, $6=$ muy útil, $7=$ =esencial, $\mathrm{TE}=$ Tutor externo, $\mathrm{Tl}=$ Tutor interno.

Aparentemente el cuestionario es más útil para guiar el desarrollo del compromiso en el trabajo, la identificación y resolución de problemas y la capacidad de indagar e investigar, pensar de forma lógica y analítica, con poder de razonamiento y análisis crítico. Una posible explicación es el hecho de que en el cuestionario de evaluación 
del practicum, lo que se utilizaron fueron ítems de aspectos que debían observarse en el alumno, de manera que observando esos ítems pudiera obtenerse una visión bastante buena del grado de consecución de una determinada capacidad. En cambio, en el cuestionario de opinión sobre la utilidad de los materiales se pregunta directamente por las competencias profesionales. Esta posible explicación refuerza la idea de la necesidad de desarrollar una guía también para los tutores externos donde se expongan todos estos aspectos de forma más explícita y detallada (qué competencias se pretenden desarrollar y evaluar y cuáles son los indicadores a través de los cuales se pretende obtener evidencia al respecto).

En lo que respecta a la utilidad de los materiales como herramienta de evaluación de las competencias profesionales (véanse Tablas 11 y 12), la opinión general es mejor que en el caso anterior. Por término medio parece que son más útiles para evaluar las competencias que para orientar su desarrollo en el estudiante, apoyando la necesidad de generar nuevos materiales explicativos para los tutores. Se aprecia además mayor grado de acuerdo en torno a los valores representativos de una opinión positiva sobre los materiales (algo útiles a esenciales).

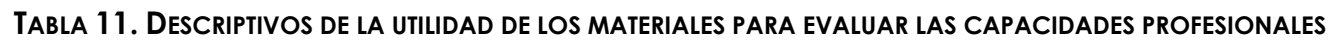
SELECCIONADAS: ESTADÍSTICOS

\begin{tabular}{|c|c|c|c|c|c|c|c|c|}
\hline & & $\mathrm{N}$ & Media & Mediana & Moda & $\begin{array}{c}\text { Desv. } \\
\text { típ. }\end{array}$ & Mín. & Máx. \\
\hline \multirow{2}{*}{$\begin{array}{c}\text { Identificación y resolución de } \\
\text { problemas }\end{array}$} & TE & 13 & 5,08 & 5,00 & 6 & 1,61 & 1 & 7 \\
\hline & $\mathrm{TI}$ & 0 & & & & & & \\
\hline \multirow{2}{*}{ Compromiso con el trabajo } & TE & 13 & 4,92 & 6,00 & 6 & 1,66 & 1 & 7 \\
\hline & $\mathrm{TI}$ & 11 & 4,18 & 5,00 & 5 & 1,94 & 1 & 7 \\
\hline \multirow{2}{*}{ Trabajo en equipo } & TE & 13 & 5,00 & 5,00 & $4^{a}$ & 1,63 & 1 & 7 \\
\hline & $\mathrm{TI}$ & 0 & & & & & & \\
\hline \multirow{2}{*}{$\begin{array}{l}\text { Indagar e investigar, pensar de } \\
\text { forma lógica y analítica, con } \\
\text { poder de razonamiento y análisis } \\
\text { crítico }\end{array}$} & TE & 13 & 4,92 & 5,00 & 6 & 1,55 & 1 & 7 \\
\hline & $\mathrm{TI}$ & 11 & 4,73 & 5,00 & 5 & 1,56 & 1 & 7 \\
\hline \multirow{2}{*}{$\begin{array}{l}\text { Aplicar los conocimientos en la } \\
\text { práctica }\end{array}$} & TE & 13 & 4,54 & 5,00 & 4 & 1,66 & 1 & 7 \\
\hline & $\mathrm{TI}$ & 11 & 5,18 & 5,00 & 5 & 1,66 & 1 & 7 \\
\hline \multirow{2}{*}{ Iniciativa } & TE & 13 & 5,15 & 6,00 & $4^{a}$ & 1,72 & 1 & 7 \\
\hline & $\mathrm{TI}$ & 11 & 3,73 & 4,00 & $3^{a}$ & 1,56 & 1 & 6 \\
\hline \multirow{2}{*}{ Aprendizaje autónomo } & TE & 12 & 4,92 & 5,50 & 6 & 1,62 & 1 & 7 \\
\hline & $\mathrm{TI}$ & 11 & 4,18 & 4,00 & 4 & 1,83 & 1 & 7 \\
\hline \multirow{2}{*}{ Análisis y síntesis } & TE & 0 & & & & & & \\
\hline & $\mathrm{TI}$ & 11 & 5,36 & 6,00 & 6 & 1,29 & 3 & 7 \\
\hline \multirow{2}{*}{ Comunicación oral y escrita } & TE & 0 & & & & & & \\
\hline & $\mathrm{TI}$ & 11 & 5,55 & 6,00 & 7 & 1,44 & 3 & 7 \\
\hline \multirow{2}{*}{ Compromiso por la calidad } & $\mathrm{TE}$ & 0 & & & & & & \\
\hline & $\mathrm{TI}$ & 11 & 4,64 & 5,00 & 6 & 1,36 & 2 & 6 \\
\hline
\end{tabular}

$\mathrm{TE}=$ Tutor externo, $\mathrm{Tl}=$ Tutor interno. ${ }^{a}$ Existen varias modas y se muestra el menor de los valores.

Para la mayoría de competencias evaluables por parte de ambos tipos de tutores, los tutores externos están más satisfechos con la utilidad de sus materiales para la evaluación que los tutores internos.

Respecto a los tutores internos, mediante sus materiales, las competencias que consideran que se pueden evaluar mejor son la capacidad de comunicación oral y 
escrita, la de análisis y síntesis, la capacidad para aplicar los conocimientos adquiridos en el máster a la práctica. Por el contrario, les resultan poco útiles para evaluar la iniciativa.

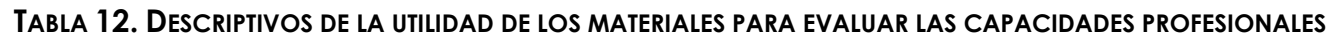
SELECCIONADAS: PORCENTAJES DE LAS RESPUESTAS DE LOS TUTORES

\begin{tabular}{|c|c|c|c|c|c|c|c|c|c|c|}
\hline CAPACIDADES & & & 1 & 2 & 3 & 4 & 5 & 6 & 7 & Total \\
\hline \multirow{4}{*}{$\begin{array}{c}\text { Identificación y resolución de } \\
\text { problemas }\end{array}$} & \multirow{2}{*}{ TE } & Frecuencia & 1 & 0 & 0 & 3 & 3 & 4 & 2 & 13 \\
\hline & & $\%$ & 7,7 & 0,0 & 0,0 & 23,1 & 23,1 & 30,8 & 15,4 & 100,0 \\
\hline & \multirow{2}{*}{$\mathrm{Tl}$} & Frecuencia & & & & & & & & \\
\hline & & $\%$ & & & & & & & & \\
\hline \multirow{4}{*}{ Compromiso con el trabajo } & \multirow{2}{*}{ TE } & Frecuencia & 1 & 0 & 1 & 3 & 1 & 6 & 1 & 13 \\
\hline & & $\%$ & 7,7 & 0,0 & 7,7 & 23,1 & 7,7 & 46,2 & 7,7 & 100,0 \\
\hline & \multirow{2}{*}{$\mathrm{Tl}$} & Frecuencia & 1 & 2 & 1 & 1 & 3 & 2 & 1 & 11 \\
\hline & & $\%$ & 9,1 & 18,2 & 9,1 & 9,1 & 27,3 & 18,2 & 9,1 & 100,0 \\
\hline \multirow{4}{*}{ Trabajo en equipo } & \multirow{2}{*}{ TE } & Frecuencia & 1 & 0 & 0 & 4 & 2 & 4 & 2 & 13 \\
\hline & & $\%$ & 7,7 & 0,0 & 0,0 & 30,8 & 15,4 & 30,8 & 15,4 & 100,0 \\
\hline & \multirow{2}{*}{$\mathrm{TI}$} & Frecuencia & & & & & & & & \\
\hline & & $\%$ & & & & & & & & \\
\hline \multirow{4}{*}{$\begin{array}{l}\text { Indagar e investigar, pensar de } \\
\text { forma lógica y analítica, con } \\
\text { poder de razonamiento y } \\
\text { análisis crítico }\end{array}$} & \multirow{2}{*}{ TE } & Frecuencia & 1 & 0 & 0 & 4 & 2 & 5 & 1 & 13 \\
\hline & & $\%$ & 7,7 & 0,0 & 0,0 & 30,8 & 15,4 & 38,5 & 7,7 & 100,0 \\
\hline & \multirow{2}{*}{$\mathrm{TI}$} & Frecuencia & 1 & 0 & 0 & 3 & 4 & 2 & 1 & 11 \\
\hline & & $\%$ & 9,1 & 0,0 & 0,0 & 27,3 & 36,4 & 18,2 & 9,1 & 100,0 \\
\hline \multirow{4}{*}{$\begin{array}{c}\text { Aplicar los conocimientos en la } \\
\text { práctica }\end{array}$} & \multirow{2}{*}{ TE } & Frecuencia & 1 & 1 & 0 & \begin{tabular}{|l|}
4 \\
\end{tabular} & 3 & 3 & 1 & 13 \\
\hline & & $\%$ & 7,7 & 7,7 & 0,0 & 30,8 & 23,1 & 23,1 & 7,7 & 100,0 \\
\hline & \multirow{2}{*}{ TI } & Frecuencia & 1 & 0 & 0 & \begin{tabular}{|l|}
1 \\
\end{tabular} & 4 & 3 & 2 & 11 \\
\hline & & $\%$ & 9,1 & 0,0 & 0,0 & 9,1 & 36,4 & 27,3 & 18,2 & 100,0 \\
\hline \multirow{4}{*}{ Iniciativa } & \multirow{2}{*}{ TE } & Frecuencia & 1 & 0 & 0 & 4 & 1 & 4 & 3 & 13 \\
\hline & & $\%$ & 7,7 & 0,0 & 0,0 & 30,8 & 7,7 & 30,8 & 23,1 & 100,0 \\
\hline & \multirow{2}{*}{$\mathrm{TI}$} & Frecuencia & 1 & 1 & 3 & 3 & 1 & 2 & 0 & 11 \\
\hline & & $\%$ & 9,1 & 9,1 & 27,3 & 27,3 & 9,1 & 18,2 & 0,0 & 100,0 \\
\hline \multirow{4}{*}{ Aprendizaje autónomo } & \multirow{2}{*}{ TE } & Frecuencia & 1 & 0 & 0 & 4 & 1 & 5 & 1 & 12 \\
\hline & & $\%$ & 8,3 & 0,0 & 0,0 & 33,3 & 8,3 & 41,7 & 8,3 & 100,0 \\
\hline & \multirow{2}{*}{$\mathrm{TI}$} & Frecuencia & 2 & 0 & 0 & 4 & 3 & 1 & 1 & 11 \\
\hline & & $\%$ & 18,2 & 0,0 & 0,0 & 36,4 & 27,3 & 9,1 & 9,1 & 100,0 \\
\hline \multirow{4}{*}{ Análisis y síntesis } & \multirow{2}{*}{ TE } & Frecuencia & & & & & & & & \\
\hline & & $\%$ & & & & & & & & \\
\hline & Tt & Frecuencia & 0 & 0 & 1 & 2 & 2 & 4 & 2 & 11 \\
\hline & 11 & $\%$ & 0,0 & 0,0 & 9,1 & 18,2 & 18,2 & 36,4 & 18,2 & 100,0 \\
\hline & & Frecuencia & & & & & & & & \\
\hline & IE & $\%$ & & & & & & & & \\
\hline Comunicacion oral y escrita & TI & Frecuencia & 0 & 0 & 1 & 2 & 2 & 2 & 4 & 11 \\
\hline & 11 & $\%$ & 0,0 & 0,0 & 9,1 & 18,2 & 18,2 & 18,2 & 36,4 & 100,0 \\
\hline & TF & Frecuencia & & & & & & & & \\
\hline mpromise por la calidad & IE & $\%$ & & & & & & & & \\
\hline compromiso por la callada & & Frecuencia & 0 & 1 & 1 & 3 & 2 & 4 & 0 & 11 \\
\hline & II & $\%$ & 0,0 & 9,1 & 9,1 & 27,3 & 18,2 & 36,4 & 0,0 & 100,0 \\
\hline
\end{tabular}

$1=$ ninguna utilidad, $2=$ muy poca utilidad, $3=$ poca utilidad, $4=$ algo útil, $5=$ bastante útil, $6=$ muy útil, $7=$ =esencial, TE= Tutor externo, $\mathrm{Tl}=$ Tutor interno. 
Esto nos lleva a pensar que, dado que este colectivo otorga poco valor a la importancia de esta capacidad y que los materiales no les resultan útiles para guiar su desarrollo y evaluarla, tal vez deberíamos replantearnos la conveniencia de que sean los tutores internos quieres valoren dicha competencia.

En opinión de los tutores de empresa, las capacidades que mejor se pueden evaluar a través del cuestionario son la iniciativa, la identificación y resolución de problemas y el trabajo en equipo. Mientras que la que peor se evalúa es la aplicación de los conocimientos a la práctica.

\section{CONCLUSIONES}

Existe un amplio consenso internacional sobre la necesidad de que la formación académica de los futuros contables y auditores incluya la formación en determinadas competencias profesionales que facilitarán su inserción laboral y mejorarán su desempeño. Si bien la propuesta de competencias profesionales está comúnmente aceptada, el debate sobre cómo se deben evaluar sigue abierto. En este trabajo proponemos utilizar el practicum, es decir, la realización de una práctica externa curricular como medio de garantizar la formación en estas competencias. Debido a las peculiaridades de esta asignatura, se debe establecer un protocolo de actuación en el cual se garantice que la formación recibida por el alumno sea la adecuada independientemente del lugar en el que realice la práctica.

Para ello, hemos diseñado una serie de materiales (documentos, procedimientos y cuestionarios) que sirvan de guía tanto al alumno, como al tutor interno y al externo del Máster en Contabilidad y Auditoría de la Universidad de Cádiz. Una vez terminado el proceso de evaluación del practicum hemos recabado y analizado la opinión de los evaluadores del practicum para determinar si dichos materiales les han sido útiles en su labor.

Los resultados obtenidos muestran que si bien existe consenso sobre las competencias que pueden ser desarrolladas y evaluadas a través de Máster, parece que los materiales propuestos no han resultado del todo útiles para los tutores internos ni externos.

Una razón que puede explicar estos resultados, en el caso de los tutores externos, es que no tuvieron en cuenta los materiales hasta que finalizó la práctica y tuvieron que llevar a cabo la evaluación, por lo que éstos no les pudieron servir de guía. Además, la comunicación entre tutor interno y externo, como herramienta para poner en común las competencias a evaluar y la metodología para hacerlo, no ha sido lo suficientemente fluida en todos los casos.

Otra posible explicación es que mientras en los cuestionarios de evaluación del practicum se utilizaron ítems o hechos observables para inferir el nivel alcanzado por los alumnos en las distintas competencias, en los cuestionarios de opinión sobre la utilidad de los materiales se preguntaba directamente por las competencias profesionales en cuestión. De manera que los resultados obtenidos podrían deberse a la redacción de los cuestionarios más que a la propia técnica en sí.

A la luz de estos resultados, la principal aportación de este trabajo es proporcionar a la comunidad universitaria una propuesta de competencias profesionales que pueden ser trabajadas a través del Máster, así como unos materiales que pueden servir para su seguimiento y evaluación. También se aporta una experiencia docente en un ámbito distinto al habitual al tener como factor diferenciador la participación de evaluadores externos. Con respecto a esto, se ha puesto de manifiesto que, siguiendo a Samkin y Francis (2008), una posible mejora que puede plantearse en el proceso de aprendizaje 
de los alumnos de esta disciplina es la realización de un portafolio como modelo de enseñanza, aprendizaje y evaluación. En este sentido, sería conveniente llevar a cabo una guía para uso de los alumnos, tutores académicos y tutores de empresa en la que se recogieran no sólo las competencias a evaluar, sino también los hechos observables que pueden permitir dicha evaluación, un itinerario de reuniones y contactos tutor interno-tutor externo, tutor externo-alumno y tutor interno-alumno que permitan consensuar y clarificar los objetivos de aprendizaje y la metodología e, incluso, los documentos o informes que deberán irse confeccionando y que asegurarán un mayor seguimiento del aprendizaje del alumno.

Dicha guía, si se pone a disposición de los tutores externos de forma anticipada, permitiría garantizar cierta homogeneidad en la evaluación de todos los alumnos del Máster independientemente del lugar en el que realizaran la práctica y el tutor de empresa que los formara. Con respecto a los tutores internos, esta guía facilitaría la comunicación con los tutores externos ya que les permitiría consensuar los objetivos y la metodología de la práctica sobre una base común. Finalmente, los alumnos agradecerán tener claras las competencias que deberán desarrollar puesto que así podrán sacar más provecho de su estancia en la empresa o entidad.

\section{BIBLIOGRAFIA}

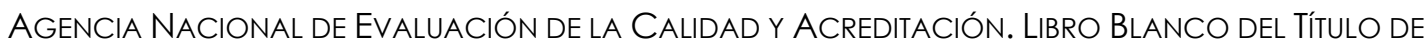
GRADO EN ECONOMÍA Y EN EMPRESA: http://www.aneca.es/media/150292/libroblanco_economia_def.pdf

ARQUERO MONTAÑo, J.L. [2000]: Capacidades no técnicas en el perfil profesional en contabilidad: las opiniones de docentes y profesionales, Revista Española de Financiación y Contabilidad, vol. 29, núm. 103: 149-172.

ARquero Montaño, J.L.; Donoso Anes, J.A.; JimÉnez CARdoso, S.M. y González González, J.M. [2009]: Análisis exploratorio del perfil demandado para administración y dirección de empresas: implicaciones para el área contable, Revista de Contabilidad, vol. 12, núm. 2: 181-214.

CANO GARCíA, E.M. [2008]: La evaluación por competencias en la educación superior, Profesorado, revista de curriculum y formación del profesorado, vol. 12, núm. 3: 1-16, http://www.ugr.es/ recfpro/rev123COL1.pdf (consultado el 5 de septiembre de 2010).

CONSEJO SOCIAL Y DIRECCIÓN GENERAL DE EMPLEO DE LA UNIVERSIDAD DE CÁDIZ [2010]: Proyectos de Implantación de un Programa de Formación y Certificación de Competencias para Titulados Universitarios, http://www.competenciaslaborales.es/

Houston, W.R. [1990]: Formación del profesorado basada en competencias (Competency-based Teacher Education, CBTE), en Husen, T. y Postlethwaite, T.N.: Enciclopedia Internacional de Educación. T. IV. Barcelona. Vicens Vives: 2717- 2727.

INTERNATIONAL FEDERATION OF ACCOUNTANTS [2009]: Handbook of International Education Pronouncements. International Federation of Accountants, febrero, http://www.ifac.org

INTERNATIONAL FEDERATION OF ACCOUNTANTS [2010]: Handbook of International Education Pronouncements. International Federation of Accountants, agosto, edición 2010, http://www.ifac.org

INTERNATIONAL FEDERATION OF ACCOUNTANTS [2010]: International Education Practice Statement (IEPS) 3 Practical Experience Requirements - Initial Professional Development for Professional Accountants, en Handbook of International Education Pronouncements, Agosto, Edición 2010, http://www.ifac.org 
KAVANAGH, M.A. y DRENNAN, L. [2008]: What skills and attributes does an accounting graduate need? Evidence from student perceptions and employer expectations, Accounting and Finance, vol. 48: pp. 279-300.

LEY ORGÁNICA 5/2002 DE 19 DE JUNIO: de las Cualificaciones y de la Formación Profesional. BOE núm. 147.

SAMKIN, G. Y FRANCIS, G. [2008]: "Introducing a learning portfolio in an undergraduate financial accounting course", Accounting Education, vol. 17, núm. 3: 233-271.

Solanes PuChOl, P.; NúÑez NúÑez, R. y Rodríguez Marín, J. [2008]: Elaboración de un cuestionario para la evaluación de competencias genéricas en estudiantes universitarios Apuntes de Psicología, vol. 26, núm. 1: 35-49.

TejadA Fernández, J. [2005]: El trabajo por competencias en el prácticum: cómo organizarlo y cómo evaluarlo. VIII Symposium Internacional sobre Practicum y Prácticas en empresas en la formación universitaria. Poio, 30 junio-2 julio. http://webs2002.uab.es/paplicada/htm/papers/Trabcompetenciaspracticum.p df (consultado el 5 de septiembre de 2009).

Villa Sánchez, A. y Poblete Ruiz, M. [2004]: Practicum y evaluación de competencias, profesorado. "Revista de curriculum y formación del profesorado", vol. 8, núm. 2:1-19. http://www.ugr.es/ recfpro/Rev82.html

Zabalza, Miguel A. [2004]: "Competencias personales y profesionales en el practicum". Documento de trabajo. Universidad de Santiago de Compostela. http://redaberta.usc.es/uvi/public_html/images/pdf2001/zabalza.pdf, (consultado 3 de septiembre de 2010). 


\section{ANEXOS}

ANEXO 1. MODELO DE MEMORIA DE PRÁCTICAS

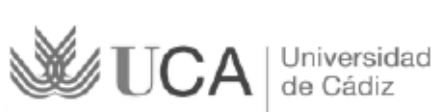

Master Oficial de Contabilidad y Auditoría

\begin{tabular}{|l|l|l|}
\hline \multicolumn{3}{|c|}{ MODELO DE MEMORIA DE PRÁCTICAS } \\
\hline NOMBRE Y APELLIDOS DEL ALUMNO/A & DNI \\
\hline TELÉFONOS & E-MAIL \\
\hline NOMBRE DE LA EMPRESA & NOMBRE DEL TUTOR/A EN LA EMPRESA \\
\hline NOMBRE DEL TUTOR/A EN LA UNIVERSIDAD & $\begin{array}{l}\text { LUGAR DE REALIZACIÓN DE LAS PRACTICAS } \\
\text { (LOCALIDAD, CÓDIGO POSTAL) }\end{array}$ \\
\hline FECHA INICIO PRÁCTICAS & FECHA TERMINACIÓN PRÁCTICAS \\
\hline \multicolumn{3}{|c|}{ TOTAL HORAS DE PRÁCTICAS REALIZADAS POR EL/LA ALUMNO/A } \\
\hline
\end{tabular}

Indicaciones sobre la presentación de la Memoria:

- Escrita:

$\Rightarrow \quad$ Se recomienda una extensión mínima de 20 folios de tamaño A4 y máxima de 40.

$\Rightarrow \quad$ Letra Arial 12

$\Rightarrow \quad$ Inclusión de Índice

$\Rightarrow \quad$ Inclusión de Bibliografía si se estima conveniente

$\Rightarrow \quad$ Inclusión de Anexos si procede
$\Rightarrow \quad$ Duración de la presentación: 30 minutos

$\Rightarrow \quad$ Número máximo de diapositivas 30.

Describir la empresa o institución, en general, y la dependencia o departamento donde ha desempeñado la práctica y personas con las que se ha relacionado durante la misma

Debe hacerse una breve descripción de las instalaciones y un organigrama esquemático de la empresa que oriente sobre la ubicación funcional del servicio o departamento en que ha realizado la práctica. Se trata de que analice cómo se organiza la toma de decisiones y la responsabilidad entre las personas que están integradas en dicha estructura. De igual forma, cabe mencionar aquí cómo son las relaciones informales en cuanto a acogida de nuevos miembros en la organización, el traslado al estudiante de la cultura de la empresa o institución, etc.

\section{Descripción del programa formativo de la práctica}

Presente de forma resumida los objetivos formativos de la práctica, las tareas o actividades en las que se han plasmados dichos objetivos y los materiales utilizados o generados.

Describir la relación entre la formación recibida en el Máster y la práctica realizada. Se trata de analizar qué conocimientos o herramientas aprendidos en el Máster han estado más relacionados con la práctica realizada y, por tanto, qué actividades o tareas realizadas en la práctica han servido de puesta en práctica de dichos conocimientos.

Fecha y firma del alumno:

Fecha y firma del tutor académico: 
Estíbaliz Biedma, Nieves Gómez, Emiliano Ruiz El practicum como herramienta de evaluación

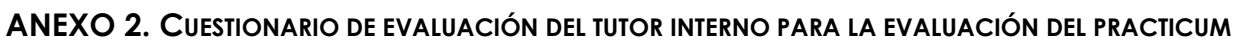

MTT $\boldsymbol{\Delta}$ | Universidad

de Cádiz

Master Oficial de Contabilidad y Auditoría

\begin{tabular}{|c|c|c|}
\hline \multicolumn{3}{|c|}{ CUESTIONARIO DE EVALUACIÓN DEL/LA TUTOR/A INTERNO/A } \\
\hline \multicolumn{3}{|l|}{ NOMBRE Y APELLIDOS DEL ALUMNO/A } \\
\hline NOMBRE DEL TUTOR/A & & CENTRO UNIVERSITARIO / TÍTULO \\
\hline \multicolumn{3}{|l|}{ NOMBRE DE LA EMPRESA } \\
\hline \multicolumn{3}{|l|}{ LÍNEAS DE FORMACIÓN DE LA PRÁCTICA } \\
\hline \multicolumn{2}{|l|}{ FECHA INICIO PRÁCTICAS } & FECHA TERMINACIÓN PRÁCTICAS \\
\hline \multicolumn{3}{|c|}{ TOTAL HORAS DE PRÁCTICAS REALIZADAS POR EL/LA ALUMNO/A } \\
\hline Número de horas semanales & Número de semanas & TOTAL \\
\hline
\end{tabular}

1. ACTIVIDADES DESARROLLADAS POR EL ALUMNO/A

-

$\cdot$

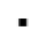

-

2. VALORACIÓN DE LAS ACTITUDES Y DEL CUMPLIMIENTO DEL ALUMNO/A

\begin{tabular}{|l|c|c|c|c|c|c|c|c|c|c|c|}
\hline \multicolumn{10}{c}{ Muy mal } \\
\hline Regularidad en la asistencia a tutorias obligatorias & 0 & 1 & 2 & 3 & 4 & 5 & 6 & 7 & 8 & 9 & 10 \\
\hline Cumplimiento de normas sobre prácticas externas & 0 & 1 & 2 & 3 & 4 & 5 & 6 & 7 & 8 & 9 & 10 \\
\hline Interés por aprender & 0 & 1 & 2 & 3 & 4 & 5 & 6 & 7 & 8 & 9 & 10 \\
\hline Motivación / participación actividades voluntarias & 0 & 1 & 2 & 3 & 4 & 5 & 6 & 7 & 8 & 9 & 10 \\
\hline Iniciativa & 0 & 1 & 2 & 3 & 4 & 5 & 6 & 7 & 8 & 9 & 10 \\
\hline Autonomía & 0 & 1 & 2 & 3 & 4 & 5 & 6 & 7 & 8 & 9 & 10 \\
\hline
\end{tabular}


Estíbaliz Biedma, Nieves Gómez, Emiliano Ruiz El practicum como herramienta de evaluación

MTT A Universidad

de Cádiz

3. VALORACIÓN DE LOS CONOCIMIENTOS Y COMPETENCIAS ADQUIRIDOS

\begin{tabular}{|c|c|c|c|c|c|c|c|c|c|c|c|}
\hline \multicolumn{10}{|c|}{ Muy mal } & \multicolumn{2}{|c|}{ Muy bien } \\
\hline Aprovechamiento de la práctica & 0 & 1 & 2 & 3 & 4 & 5 & 6 & 7 & 8 & 9 & 10 \\
\hline Valoración de la memoria presentada & 0 & 1 & 2 & 3 & 4 & 5 & 6 & 7 & 8 & 9 & 10 \\
\hline
\end{tabular}

4. VALORACIÓN GLOBAL DEL ALUMNO:

Evalúe el desempeño de la práctica por parte del alumno/a

Muy mal
\begin{tabular}{|c|c|c|c|c|c|c|c|c|c|c|}
\hline 0 & 1 & 2 & 3 & 4 & 5 & 6 & 7 & 8 & 9 & 10 \\
\hline
\end{tabular}

5. VALORACIÓN DE LA EMPRESA Y EL TUTOR EXTERNO:

Valore el nivel formativo de la práctica para el alumno/a

Valore el cumplimiento de las líneas de formación por parte de la empresa

Valore las condiciones de espacio y materiales que la empresa ha puesto a disposición del alumno/a

Valore la relación que el tutor de empresa ha tenido con el alumno/a

Muy mal
\begin{tabular}{|c|c|c|c|c|c|c|c|c|c|c}
\hline 0 & 1 & 2 & 3 & 4 & 5 & 6 & 7 & 8 & 9 & 10 \\
\hline 0 & 1 & 2 & 3 & 4 & 5 & 6 & 7 & 8 & 9 & 10 \\
\hline 0 & 1 & 2 & 3 & 4 & 5 & 6 & 7 & 8 & 9 & 10 \\
\hline 0 & 1 & 2 & 3 & 4 & 5 & 6 & 7 & 8 & 9 & 10 \\
\hline
\end{tabular}

\section{SATISFACCIÓN GENERAL CON LA PRÁCTICA:}

Satisfacción general con la práctica (gestión de la práctica, \begin{tabular}{|l|l|l|l|l|l|l|l|l|l|l} 
Muy mal \\
condiciones en la empresa, relaciones con el tutor externo, \\
desempeño del alumno, etc.):
\end{tabular}

7. INCIDENCIAS A DESTACAR:

\section{OBSERVACIONES COMPLEMENTARIAS:}


Estíbaliz Biedma, Nieves Gómez, Emiliano Ruiz El practicum como herramienta de evaluación

ANEXO 3. Cuestionario del tUtor eXterno para la eVAlUACión del practicum

MTTCAniversidad

de Cádiz

Master Oficial de Contabilidad y Auditoría

CUESTIONARIO DE EVALUACIÓN DEL/LA TUTOR/A EXTERNO/A

\begin{tabular}{|l|l|l|}
\hline \multicolumn{2}{|l|}{ NOMBRE Y APELLIDOS DEL ALUMNO/A } & DNI \\
\hline NOMBRE DE LA EMPRESA & $\begin{array}{l}\text { DEPARTAMENTO / ÁREA DE REALIZACIÓN DE LA } \\
\text { PRÁCTICA }\end{array}$ \\
\hline NOMBRE DEL TUTOR/A EN LA EMPRESA & CARGO EN LA EMPRESA \\
\hline \multicolumn{2}{|c|}{ HECHA INICIO PRÁCTICAS Horas de prácticas } \\
\hline \multicolumn{1}{|c|}{ FECHA TERMINACIÓN PRÁCTICAS } \\
\hline Horas semanales & Número de semanas & TOTAL \\
\hline
\end{tabular}

1. ACTIVIDADES DESARROLLADAS POR EL ALUMNO/A

-

2. VALORACIÓN DEL CUMPLIMIENTO

\begin{tabular}{|c|c|c|c|c|c|c|c|c|c|c|c|}
\hline \multicolumn{10}{|c|}{ Muy mal } & \multicolumn{2}{|c|}{ Muy bien } \\
\hline Regularidad en la asistencia & 0 & 1 & 2 & 3 & 4 & 5 & 6 & 7 & 8 & 9 & 10 \\
\hline Puntualidad y cumplimiento de horarios & 0 & 1 & 2 & 3 & 4 & 5 & 6 & 7 & 8 & 9 & 10 \\
\hline Conocimiento de normas y usos de la empresa o institución & 0 & 1 & 2 & 3 & 4 & 5 & 6 & 7 & 8 & 9 & 10 \\
\hline Respeto a la confidencialidad & 0 & 1 & 2 & 3 & 4 & 5 & 6 & 7 & 8 & 9 & 10 \\
\hline
\end{tabular}

3. VALORACIÓN DE HABILIDADES Y COMPETENCIAS PROFESIONALES

\begin{tabular}{|l|c|c|c|c|c|c|c|c|c|c|c|c|}
\multicolumn{10}{c}{ Muy mal } \\
\hline Adaptación al centro de trabajo & 0 & 1 & 2 & 3 & 4 & 5 & 6 & 7 & 8 & 9 & 10 \\
\hline Capacidad de trabajo en equipo & 0 & 1 & 2 & 3 & 4 & 5 & 6 & 7 & 8 & 9 & 10 \\
\hline Capacidad de analizar y resolver problemas & 0 & 0 & 1 & 2 & 3 & 4 & 5 & 6 & 7 & 8 & 9 & 10 \\
\hline Responsabilidad & 0 & 1 & 2 & 3 & 4 & 5 & 6 & 7 & 8 & 9 & 10 \\
\hline Capacidad de aplicación de conocimientos & 0 & 1 & 2 & 3 & 4 & 5 & 6 & 7 & 8 & 9 & 10 \\
\hline Sentido crítico & 0 & 1 & 2 & 3 & 4 & 5 & 6 & 7 & 8 & 9 & 10 \\
\hline
\end{tabular}


Estíbaliz Biedma, Nieves Gómez, Emiliano Ruiz El practicum como herramienta de evaluación

4. VALORACIÓN DE LAS ACTITUDES

\begin{tabular}{|l|c|c|c|c|c|c|c|c|c|c|c|}
\hline Muy mal \\
\hline Interés por actividades / por aprender & 0 & 1 & 2 & 3 & 4 & 5 & 6 & 7 & 8 & 9 & 10 \\
\hline Motivación / participación actividades voluntarias & 0 & 1 & 2 & 3 & 4 & 5 & 6 & 7 & 8 & 9 & 10 \\
\hline Iniciativa & 0 & 1 & 2 & 3 & 4 & 5 & 6 & 7 & 8 & 9 & 10 \\
\hline Corrección en el trato & 0 & 1 & 2 & 3 & 4 & 5 & 6 & 7 & 8 & 9 & 10 \\
\hline Autonomía & 0 & 1 & 2 & 3 & 4 & 5 & 6 & 7 & 8 & 9 & 10 \\
\hline
\end{tabular}

5. APORTACIÓN DEL/LA ALUMNO/A A LA EMPRESA:

6. INCIDENCIAS A DESTACAR:

\section{VALORACIÓN GLOBAL DEL ALUMNO/A:}

Valore la práctica realizada por el/la alumno/a en su conjunto

Muy mal
\begin{tabular}{|c|c|c|c|c|c|c|c|c|c|c|}
\hline 0 & 1 & 2 & 3 & 4 & 5 & 6 & 7 & 8 & 9 & 10 \\
\hline
\end{tabular}

\section{SATISFACCIÓN GENERAL CON LA PRÁCTICA:}

Mutisfacción general con la práctica (gestión de la práctica, $\begin{aligned} & \text { Mul } \\ & \text { formación en la empresa, relaciones con el tutor interno, } \\ & \text { desempeño del alumno, etc.): }\end{aligned}$

9. OBSERVACIONES COMPLEMENTARIAS: 


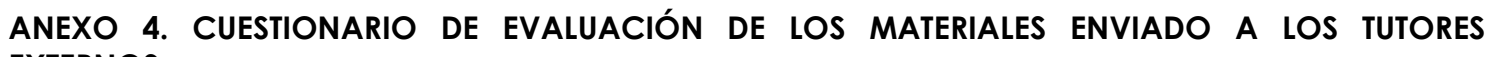
EXTERNOS.

\section{BLOQUE I. CUESTIONES BIOGRÁFICAS}

\begin{tabular}{|l|}
\hline Edad: \\
\hline Sexo: $\quad \square$ Hombre $\square$ Mujer \\
\hline Nivel de estudios: $\quad$ Doctor $\quad \square$ Licenciado $\quad \square$ Diplomado \\
\hline Experiencia laboral ( ${ }^{\circ}$ de años): \\
\hline Tamaño de la empresa ( $n^{\circ}$ de trabajadores): \\
\hline Actividad empresarial: \\
\hline
\end{tabular}

\section{BLOQUE II. CAPACIDADES PROFESIONALES}

Según su experiencia, nos gustaría saber la importancia que tienen las siguientes capacidades para el adecuado desempeño de las tareas propias de un titulado. Valore de 1 a 7 ( $1=$ ninguna importancia, 2= muy poca importancia, 3= poca importancia, 4= algo importante, $5=$ bastante importante, $6=$ muy importante, $7=$ =esencial).

\begin{tabular}{|l|l|}
\hline Capacidades & Importancia \\
\hline Identificación y resolución de problemas & \\
\hline Compromiso con el trabajo & \\
\hline Trabajo en equipo & \\
\hline $\begin{array}{l}\text { Indagar e investigar, pensar de forma lógica y analítica, con poder de } \\
\text { razonamiento y análisis crítico. }\end{array}$ & \\
\hline Aplicar los conocimientos en la práctica & \\
\hline Iniciativa & \\
\hline Aprendizaje autónomo & \\
\hline
\end{tabular}

Si quiere realizar algún comentario adicional sobre cualquiera de las capacidades, $u$ otras no incluidas, puede hacerlo a continuación:

\section{BLOQUE III. UTILIDAD DE LOS MATERIALES}

Según su experiencia, nos gustaría saber en qué medida la utilización del cuestionario le ha servido de guía para el desarrollo de las siguientes capacidades profesionales.

Igualmente, valore, en la segunda columna en qué medida la utilización del cuestionario le ha servido de guía para la evaluación de las siguientes capacidades profesionales. 
Estíbaliz Biedma, Nieves Gómez, Emiliano Ruiz El practicum como herramienta de evaluación

( 1 =ninguna utilidad, 2= muy poca utilidad, 3= poca utilidad, 4= algo útil, 5= bastante útil, $6=$ muy útil, $7=$ =sencial).

\begin{tabular}{|l|l|l|}
\hline \multicolumn{1}{|c|}{ Capacidades } & Desarrollo & Evaluación \\
\hline Identificación y resolución de problemas & & \\
\hline Compromiso con el trabajo & & \\
\hline Trabajo en equipo & & \\
\hline Indagar e investigar, pensar de forma lógica y analítica, con & & \\
\hline Aplicar los conocimientos en la práctica & & \\
\hline Iniciativa & & \\
\hline Aprendizaje autónomo & & \\
\hline
\end{tabular}

En caso de haber asignado a la utilidad del cuestionario valores entre 1 y 3, le rogamos que exponga las principales causas a continuación:

Si quiere realizar alguna sugerencia sobre posibles herramientas de utilidad en el desarrollo y evaluación de competencias profesionales en la empresa, puede hacerlo a continuación:

Agradecemos enormemente la ayuda que nos ha prestado en esta investigación.

Si tiene interés en conocer los resultados de este trabajo indíquelo y le mandaremos el informe a su dirección de correo electrónico.

Muchas gracias. 
ANEXO 5. CUESTIONARIO DE EVALUACIÓN DE LOS MATERIALES ENVIADO A LOS TUTORES INTERNOS.

\section{BLOQUE I. CUESTIONES BIOGRÁFICAS}

Experiencia docente ( $n^{\circ}$ de años):

¿Has tenido experiencia laboral fuera de la universidad?

$\square$ Si $\quad N^{\circ}$ de años: $\quad$ Actividad empresarial:

$\square$ No

BLOQUE II. CAPACIDADES PROFESIONALES

Según tu opinión, nos gustaría saber la importancia que tienen las siguientes capacidades para el adecuado desempeño de las tareas propias de un titulado en el Máster en Contabilidad y Auditoría. Valora de 1 a 7 (1=ninguna importancia, 2= muy poca importancia, 3= poca importancia, 4= algo importante, 5= bastante importante, $6=$ muy importante, $7=$ =esencial).

\begin{tabular}{|l|l|}
\hline \multicolumn{1}{|c|}{ Capacidades } & Importancia \\
\hline Análisis y síntesis & \\
\hline Comunicación oral y escrita & \\
\hline $\begin{array}{l}\text { Pensar de forma lógica y analítica, con poder de razonamiento y } \\
\text { análisis crítico. }\end{array}$ & \\
\hline Compromiso en el trabajo & \\
\hline Iniciativa & \\
\hline Aprendizaje autónomo & \\
\hline Compromiso por la calidad & \\
\hline Aplicación de los conocimientos en la práctica & \\
\hline
\end{tabular}

Si quieres realizar algún comentario adicional sobre cualquiera de las capacidades, u otras no incluidas, a continuación: 


\section{BLOQUE III. UTILIDAD DE LOS MATERIALES}

Según tu experiencia, nos gustaría saber en qué medida la utilización de los materiales citados anteriormente te han servido de guía para el desarrollo de las siguientes capacidades profesionales.

Igualmente valora, en la segunda columna, en qué medida la utilización de estos materiales te ha servido de guía para la evaluación de las siguientes capacidades profesionales.

( 1 =ninguna utilidad, $2=$ muy poca utilidad, $3=$ poca utilidad, $4=$ algo útil, $5=$ bastante útil, $6=$ muy útil, $7=$ esencial).

\begin{tabular}{|l|l|l|}
\hline \multicolumn{1}{|c|}{ Capacidades } & Desarrollo & Evaluación \\
\hline Análisis y síntesis & & \\
\hline Comunicación oral y escrita & & \\
\hline $\begin{array}{l}\text { Pensar de forma lógica y analítica, con poder de } \\
\text { razonamiento y análisis crítico. }\end{array}$ & & \\
\hline Compromiso en el trabajo & & \\
\hline Iniciativa & & \\
\hline Aprendizaje autónomo & & \\
\hline Compromiso por la calidad & & \\
\hline Aplicación de los conocimientos en la práctica & & \\
\hline
\end{tabular}

En caso de haber asignado a la utilidad del cuestionario valores entre 1 y 3 , te rogamos que expongas a continuación las principales causas:

Si quieres realizar alguna sugerencia sobre posibles herramientas de utilidad en el desarrollo y evaluación de competencias profesionales en la práctica en empresa, puedes hacerlo a continuación:

Agradecemos enormemente tu ayuda.

Muchas gracias. 